MATHEMATICS OF COMPUTATION

Volume 67, Number 223, July 1998, Pages 1023-1046

S 0025-5718(98)00945-4

\title{
CHAOS IN THE LORENZ EQUATIONS: A COMPUTER ASSISTED PROOF. PART II: DETAILS
}

\author{
KONSTANTIN MISCHAIKOW AND MARIAN MROZEK
}

\begin{abstract}
Details of a new technique for obtaining rigorous results concerning the global dynamics of nonlinear systems is described. The technique combines abstract existence results based on the Conley index theory with rigorous computer assisted computations. As an application of these methods it is proven that for some explicit parameter values the Lorenz equations exhibit chaotic dynamics.
\end{abstract}

\section{INTRODUCTION}

In [13] an outline of a computer assisted proof that the Lorenz equations,

$$
\left\{\begin{array}{l}
\dot{x_{1}}=s\left(x_{2}-x_{1}\right), \\
\dot{x_{2}}=R x_{1}-x_{2}-x_{1} x_{3}, \\
\dot{x_{3}}=x_{1} x_{2}-q x_{3},
\end{array}\right.
$$

contain chaotic dynamics for a prescribed open set of parameter values was presented. In this paper we provide the details of this proof.

Recall that if $X$ is a metric space, $f: X \rightarrow X$ is a homeomorphism and $N \subset X$, then the maximal invariant set of $N$ is defined by $\operatorname{Inv}(N, f)=\{x \in N \mid \forall n \in$ $\left.\mathbb{Z}, f^{n}(x) \in N\right\}$.

The precise statement of the theorem we prove is as follows

Theorem 1.1. Let $P:=\left\{\left(x_{1}, x_{2}, x_{3}\right) \mid x_{3}=53\right\}$. For all parameter values in a sufficiently small neighborhood of $(s, R, q)=(45,54,10)$, there exists a Poincaré section $N \subset P$ such that the Poincaré map $g$ induced by (1) is Lipschitz and well defined. Furthermore, there exists a $d \in \mathbb{N}$ and a continuous surjection $\rho$ : $\operatorname{Inv}(N, g) \rightarrow \Sigma_{2}$ such that $\rho \circ g^{d}=\sigma \circ \rho$ where $\sigma: \Sigma_{2} \rightarrow \Sigma_{2}$ is the full shift dynamics on two symbols.

Since the full shift dynamics on two symbols is equivalent to the dynamics of the Smale horseshoe [26], this theorem implies that for some iterate the dynamics of the Poincaré map $g$ is at least as complicated as that of the horseshoe.

On the one hand, this theorem comes as no surprise. Simple and standard integration of the Lorenz equations at the parameter values $(45,54,10)$ strongly suggests the existence of horseshoe dynamics of the Poincaré map in the rectangle

Received by the editor August 11, 1995 and, in revised form, April 12, 1996 and February 18, 1997.

1991 Mathematics Subject Classification. Primary 58F13, 54H20, 65L99, 34-04, 68T15.

Research of the first author was supported in part by NSF grant 9302970 .

Research of the second author was supported by KBN, Grant 0449/P3/94/06. 


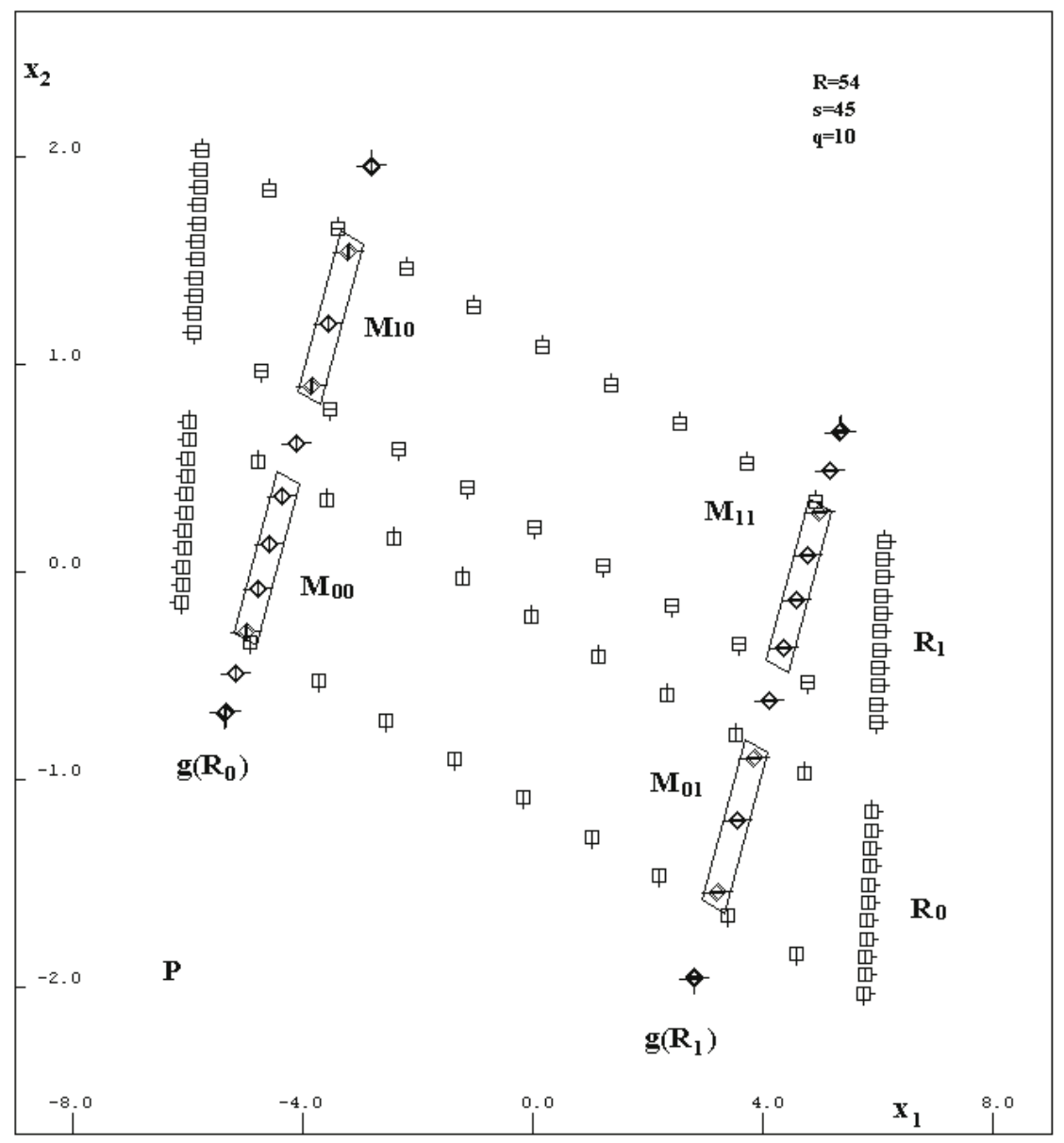

Figure 1. Numerical indication of the existence of horseshoe dynamics

$[-8,8] \times[-6,6]$ on the Poincaré plane $P$. Indeed, let $R_{0}, R_{1}$ be the two parallelograms on the plane $P$ indicated schematically in Figure 1 by a set of small marked squares in their boundary. Integrating numerically from the centers of the squares one obtains the set of points pointed out with small diamonds and corresponding marks. One can notice that the bottom side of $R_{1}$ is strongly compressed and mapped to the right bottom diamond and the top side of $R_{1}$ is mapped to the right top diamond. Similarly, the bottom and top sides of $R_{2}$ are mapped respectively to the left bottom and top diamonds. Thus, Figure 1 provides an almost textbook picture of the G-horseshoe. Unfortunately, this simple integration cannot provide us with the constants of hyperbolic expansion and contraction, nor the geometric [26] or analytic [16] transversality conditions which are necessary to prove the existence of a conjugacy with a horseshoe.

What is perhaps more surprising, given the central role that the Lorenz equations have played in the development of the theory of strange attractors, is that this theorem is, to the best of our knowledge, the first rigorous demonstration of the existence of chaotic dynamics for this system of ordinary differential equations. Since the announcement of our result, two independent proofs demonstrating the 
existence of chaotic dynamics for the Lorenz equations have appeared. The first, which is, also, a computer assisted proof is due to B. Hassard, S. Hastings, W. Troy, and J. Zhang [5] and is, in spirit, quite similar to our result. An abstract topological condition (obtained by Hastings and Troy [6]) which guarantees the existence of shift dynamics is verified using interval arithmetic. It should be noted that the abstract theorem is essentially a "shooting" method, and hence, on the topological level involves a question of connectedness. The advantage of this approach is twofold: first, the level of topological machinery required is minimal and second, the required computation time is small. The disadvantage is that these methods do not lend themselves to generalizations for problems in which the topological constraints are more subtle as is typically the case in higher dimensional settings. The second rigorous result is due to X.-Y. Chen [2] who analytically demonstrated the existence of a transverse homoclinic point within a certain range of parameter values. Obviously, this is a highly advantageous result. Unfortunately, it depends on estimates which are not valid for all parameter ranges, in particular, those of this paper. This difficulty is, of course, not unique to Chen. In fact, obtaining the necessary estimates is the central obstacle for most of the non-linear analysis. In both the result we report here and that of [5] the computer was used to overcome this obstacle.

This last comment should raise questions. After all, it is absolutely clear that computers can be used to obtain estimates and, as was alluded to earlier, the geometric or analytic estimates required to guarantee the existence of a horseshoe are well known. Why wasn't the computer used much earlier to obtain such results? The difficulty is that in order to obtain conjugacy results it is necessary to have estimates not only along orbits, but also on the derivatives along the orbits. This results in a tremendous demand on computing resources. An attempt to prove chaos in the Lorenz equations via checking in a numerical computation the existence of a transverse homoclinic point was undertaken by H. Spreuer and E. Adams in [27]. The authors have found the power of the present day computers not sufficient to perform this task rigorously. A successful computation of this type but for different equations has been carried out recently by T. Rage, A. Neumaier, and C. Schlier [23]. The cost of their computation was 2000 hours CPU time on an IBM 3090-18.

The difficulty in the rigorous numerical treatment of chaos in the Lorenz equations lies in the fact that the chaotic set is located very close to the stable manifold of the origin, where the Poincaré map is discontinuous. As a consequence the Poincaré map exhibits expansion which goes to infinity when the stable manifold is approached. This causes an unusually strong wrapping effect (see [21]). A relatively strong compression in another direction is not very helpful, because it always stays bounded.

To overcome this problem we introduce the technique of intermediate sections, which resembles the multiple shooting used in the numerical treatment of boundary value problems (see [1]). However, our technique is also substantially different. The most important difference is that it is not localized to an area around one trajectory like in the BVP, which causes that several technical difficulties have to be overcome to guarantee that the intermediate Poincaré maps are well defined.

The technique let us perform the necessary computations in 33 hours CPU time of a SUN SPARC 10 workstation. Let us mention that recently P. Zgliczyński (see [28]) performed a similar proof of chaos in the Rössler equations, where the Poincaré mapping is continuous and therefore there are no problems with extremely strong 
wrapping. This let him complete the necessary computations in time similar to ours but without the need of using the intermediate sections technique.

Obviously at this early stage our approach should be treated only as an indication that there is an alternative and possibly very fruitful road towards successful rigorous numerics of dynamical systems. Definitely the approach is very far from being optimized. The employment of advanced numerical techniques like the backward error analysis, the Lohner algorithm [8] or an improvement on logarithmic norms [22] should significantly speed up the proposed approach. There is also a significant potential for improving the algorithms via better utilization of the theory we use. We go in that direction in [15], where stronger results, obtained with essentially smaller computational effort and in particular for the classical case of the Lorenz equations $(R=28, s=10, q=8 / 3)$ will be presented.

Another important aspect of the techniques which will be reported on in this paper is that in principle they are applicable to a wide range of problems in dynamics (not only chaos) since they are based on Conley index theory [3], [24], [10], [11]. Moreover, the reader should not be misled by the fact that the Poincaré map studied in this paper is two-dimensional. On the theoretical level there are no constraints due to the dimension of the problem. In particular the Conley index theory is applicable even in an infinite dimensional setting.

To summarize this discussion, while we feel that Theorem 1.1 is of interest in itself, the main purpose of this paper is to attract the attention of readers to a new technique for obtaining rigorous results concerning the global dynamics of nonlinear systems. There are at least three new aspects of this technique. It applies to concrete differential equations, it can provide a relatively strong description of global dynamics (in terms of semiconjugacies), and the necessary computer assisted computations are small enough to be performed on currently available computers.

\section{An outLine}

The goal of our computations is the verification of the hypothesis of an abstract algebraic topological result, since this result guarantees the existence of chaotic dynamics. However, the emphasis of this paper is on the computational aspects of the problem. Thus, for many of the points we hope to express, an understanding of the Conley index theory is not necessary. Having said this it is, nevertheless, perhaps best to start our discussion by recalling the fundamental topological result which needs to be verified. We begin with the briefest of reviews of the Conley index theory for maps as developed in [17].

A compact set $N$ is an isolating neighborhood if $\operatorname{Inv} N \subset \operatorname{int} N$. If $N$ is an isolating neighborhood, then the set Inv $N$ is an isolated invariant set. A pair $P=\left(P_{1}, P_{2}\right)$ of compact subsets of $N$ is called an index pair in $N$ if $P_{i} \cap f^{-1}(N) \subset$ $f^{-1}\left(P_{i}\right)$ for $i=1,2, P_{1} \backslash f^{-1}(N) \subset P_{2}$ and $\operatorname{Inv} N \subset \operatorname{int}\left(P_{1} \backslash P_{2}\right)$. The map $f$ induces an endomorphism $I_{P}: H^{*}\left(P_{1}, P_{2}\right) \rightarrow H^{*}\left(P_{1}, P_{2}\right)$ of the Alexander-Spanier cohomology of $\left(P_{1}, P_{2}\right)$ (see [17]). The cohomological Conley index of an isolating neighborhood $N$ under $f$ is given by $\operatorname{Con}^{*}(N, f):=\left(C H^{*}(N, f), \chi^{*}(N, f)\right)$, where $C H^{*}(N, f)$ is the graded module obtained by quotienting $H^{*}\left(P_{1}, P_{2}\right)$ by the generalized kernel of $I_{P}$ and $\chi^{*}(N, f)$ is the induced graded module automorphism on $C H^{*}(N, f)$. One can show (see [17]) that this construction does not depend on the choice of the index pair.

Turning now to the special setting which is of interest for this paper, let $N=$ 
$N_{0} \cup N_{1}$ be an isolating neighborhood under $f$ where $N_{0}$ and $N_{1}$ are disjoint compact sets. For $k, l=0,1$, let $M_{k l}=N_{k} \cap f\left(N_{l}\right)$. Let $N_{l k}:=M_{k k} \cup M_{k l} \cup M_{l l}$. The following result is a reformulation of a special case of [12], Theorem 2.3 (cf. also [18]).

Theorem 2.1. Assume $M_{i j}$ for $i, j \in\{0,1\}$ are disjoint compact sets such that $N:=M_{00} \cup M_{01} \cup M_{10} \cup M_{11}$ is an isolating neighborhood under $f$. Furthermore assume that $N_{i}:=M_{i 0} \cup M_{i 1}$ satisfy

$$
\begin{gathered}
N \cap f\left(N_{i}\right) \subset M_{0 i} \cup M_{1 i}, \\
\operatorname{Con}^{n}\left(N_{k}, f\right)= \begin{cases}(\mathbb{Q}, \text { id }) & \text { if } n=1, \\
0 & \text { otherwise, }\end{cases}
\end{gathered}
$$

and $\chi^{*}\left(N_{l k}, f\right)$ is not conjugate to $\chi^{*}\left(N_{k}, f\right) \oplus \chi^{*}\left(N_{l}, f\right)$. Then, there exists a $d \in \mathbb{N}$ and a continuous surjection $\rho: \operatorname{Inv}(N, f) \rightarrow \Sigma_{2}$ such that $\rho \circ f^{d}=\sigma \circ \rho$ where $\sigma: \Sigma_{2} \rightarrow \Sigma_{2}$ is the full shift dynamics on two symbols.

The idea of the computer assisted proof proposed in this paper rests on verifying the assumptions of this theorem when applied to the Poincaré map $g$ and the four parallelograms $M_{00}, M_{01}, M_{10}, M_{11}$ on the plane $P$ indicated in Figure 1 by solid lines. To an individual schooled only in algebraic topology it is clear that when Theorem 2.1 applies, its hypothesis must be numerically verifiable. The reason for this is the simplicial approximation theorem [20], which states that the cohomology of a reasonable space and any reasonable continuous map can be computed via a piecewise linear approximation of the space and map. Unfortunately, the simplicial approximation theorem appears to be extremely inefficient to implement computationally. For this reason we turned to the theory of multivalued maps.

The assumptions of Theorem 2.1 make sense, also, for multivalued maps and, more importantly, if they are satisfied for a given multivalued map, then they are also satisfied for any selector of that map (a single valued map enclosed by the multivalued map). A more intuitive restatement of this comment might be as follows. The Conley index is invariant under continuous perturbations. More precisely, given $\operatorname{Con}^{*}(N, f)$, there exists $\epsilon>0$ such that if $g$ differs from $f$ by less than $\epsilon$ in the $C^{0}$ topology, then $\operatorname{Con}^{*}(N, f)=\operatorname{Con}^{*}(N, g)$. Let us now define a multivalued map $F$ by $F(x)=B_{\epsilon}(f(x))$. Obviously, if $h$ is a selector function for $F$ i.e., $h(x) \in F(x)$ for all $x$, then $N$ is an isolating neighborhood under $h$ and $\operatorname{Con}^{*}(N, h)=\operatorname{Con}^{*}(N, f)$.

Thus, to prove Theorem 1.1 it is sufficient to verify the assumptions of Theorem 2.1 for a well chosen multivalued map. Of special interest are these multivalued maps which admit some finite coding, because then the verification of the assumptions reduces to a finite (combinatorial) computation. And exactly such maps are provided by numerical methods combined with rigorous error bounds.

The procedure is roughly as follows. The domain of a single valued Lipschitz map $f$ is covered by a finite number of compact sets. A point is selected in each set and an appropriate numerical method is taken to find a ball where the value is located. Then, a Lipschitz constant of the map is used to get a larger ball enclosing the image of each compact set in the covering. Finally, the required multivalued map $F$ at a given point $x$ is defined as the intersection of the enclosures of all sets in the covering to which the point $x$ belongs. Obviously, the map obtained this way admits finite coding. It is also lower semicontinuous, convex-valued and $f$ is a selector of $F$. In such a situation we will also say that $F$ is a multivalued representation of $f$. 


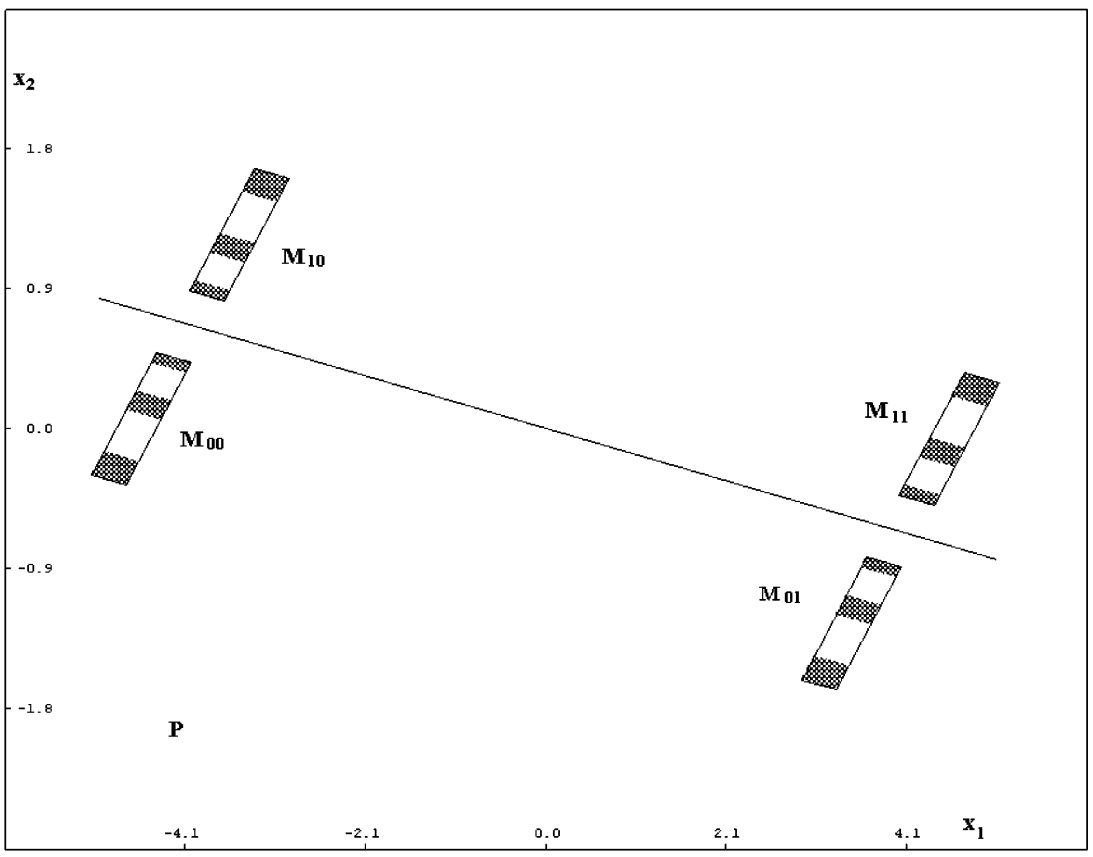

Figure 2. An index pair for $N=M_{00} \cup M_{01} \cup M_{10} \cup M_{11}$. $P_{1}=$ $N$ and $P_{2}$ is the shaded area. The line shows schematically the intersection of the stable manifold of the origin with the Poincaré plane $P$

As one can guess (see Section 3 for details), checking if a given set is an isolating neighborhood for a given multivalued representation of $f$ and finding an associated index pair is a purely combinatorial task. Thus it also may be performed rigorously by computer. Figure 2 shows an index pair $\left(P_{1}, P_{2}\right)$ obtained this way for the Poincaré map $g$ and the isolating neighborhood $N=M_{00} \cup M_{01} \cup M_{10} \cup M_{11}$. Of course, one cannot expect that an arbitrary multivalued representation of the Poincare map will fulfill the required assumptions. In fact, the smaller the values, the larger is the chance that the assumptions will be satisfied.

The maximal diameter of the values of $F$ (the size of $F$ ) is determined by the bounds on the following factors:

(1) the rounding errors,

(2) errors introduced by the numerical procedure,

(3) the Lipschitz constant.

Bounds, bad or with luck good, of all the above factors may be relatively easily found for the $t$-translation operator of the flow generated by a differential equation. The easiest way to find a multivalued representation of a Poincaré map is to estimate from the representations of the $t$-translation operators the point of intersection of the trajectory with the cross-section. This introduces the fourth factor influencing the final size of the representation of the Poincaré map.

Finding a representation of the Poincaré map with sufficiently small size may mean an enormous amount of computations in general. One of the purposes of this paper is to show that at least for some interesting problems the task of finding a 
representation of the Poincaré map with sufficiently small size may be relatively easily achieved with the help of presently available personal computers.

\section{Preliminaries}

We denote the sets of reals, nonnegative reals, integers, nonnegative integers, natural numbers and rationals by $\mathbb{R}, \mathbb{R}^{+}, \mathbb{Z}, \mathbb{Z}^{+}, \mathbb{N}, \mathbb{Q}$, respectively. Reals and integers supplemented by negative and positive infinity will be denoted by $\overline{\mathbb{R}}, \overline{\mathbb{Z}}$ respectively. For any set $X$ the notation $\mathcal{P}(X)$ will stand for the family of all subsets of $X$. A map $F: X \rightarrow \mathcal{P}(Y)$, where $X, Y$ are two arbitrary sets, will be called a multivalued map from $X$ to $Y$. The set $\operatorname{dom} F:=\{x \in X \mid F(x) \neq \emptyset\}$ is called the domain of $F$. For $A \subset X, B \subset Y$ we define the image of $A$ under $F$ by $F(A):=\bigcup\{F(x) \mid x \in A\}$, the weak preimage of $B$ under $F$ by $F^{*-1}(B):=\{x \in X \mid F(x) \cap B \neq \emptyset\}$ and the strong preimage of $B$ under $F$ by $F^{-1}(B):=\{x \in \operatorname{dom} F \mid F(x) \subset B\}$. In what follows we will use multivalued maps both in the topological setting and in a purely set theoretical setting. In the first case we assume that $X, Y$ are metric spaces and we say that $F$ is upper semicontinuous (usc) if $F^{-1}(U)$ is open for any open set $U \subset Y$ and $F$ is lower semicontinuous (lsc) if the set $F^{*-1}(U)$ is open for any open set $U \subset Y$. For $N \subset \operatorname{dom} F$ we put $\operatorname{diam}_{N} F:=\sup \{\operatorname{diam} F(x) \mid x \in N\}$. The diameter of $F$ over its domain will be called its size.

If $X$ is a metric space and $A \subset X$, then we denote the boundary, the interior and the closure of $A$ respectively by bd $A, \operatorname{int} A$ and $\operatorname{cl} A$.

Let $\varphi: \mathbb{R} \times X \rightarrow X$ be a flow. Essentially, in this paper we restrict our attention to $X=\mathbb{R}^{3}$ but since several lemmas are purely topological and may turn out to be useful in other applications we begin with the general setting. For $x \in X$ we will denote the trajectory (positive trajectory) of $x$ by $\varphi(x),\left(\varphi^{+}(x)\right)$. If $y \in \varphi^{+}(x)$, then there is a minimal non-negative $t \in \mathbb{R}^{+}$such that $y=\varphi(t, x)$. We will denote it by $t(x, y)$. The notation $[x, y]$ will stand for the set $\{\varphi(t, x) \mid 0 \leq t \leq t(x, y)\}$. For a fixed $t>0$ the map $\varphi_{t}: X \ni x \rightarrow \varphi(x, t) \in X$ will be called the $t$-translation operator of $\varphi$.

If $X=\mathbb{R}^{d}$, then we assume that the flow $\varphi$ is induced by a differential equation

$$
\dot{x}=V(x),
$$

where $V: \mathbb{R}^{d} \rightarrow \mathbb{R}^{d}$ is a $C^{1}$-vector field on $\mathbb{R}^{d}$. Let $\|\|:. \mathbb{R}^{d} \rightarrow \mathbb{R}^{+}$be a fixed norm on $\mathbb{R}^{d}$. For $x \in \mathbb{R}^{d}, A \subset \mathbb{R}^{d}$ and $r>0$ we put $\operatorname{dist}(x, A):=\sup \{\|x-y\| \mid y \in A\}$, $B(A, r):=\left\{y \in \mathbb{R}^{d} \mid \operatorname{dist}(y, A) \leq r\right\}$ and $B(x, r):=B(\{x\}, r)$. We will mark the distance and the ball with the sup subscript when they relate to the supremum norm given by $\|x\|_{\text {sup }}:=\max \left\{\left|x_{i}\right| \mid i=1, \ldots, d\right\}$. If $A: \mathbb{R}^{d} \rightarrow \mathbb{R}^{d}$ is a linear map, then $\|A\|:=\sup \left\{\|A x\| \mid x \in \mathbb{R}^{d},\|x\| \leq 1\right\}$ will denote the operator norm of $A$.

\section{DisCRETIZATION OF SPACE AND MAPS}

Our aim now is to show that the assumptions of Theorem 2.1 may be translated to the language of multivalued maps which admit some finite description. The first step is the construction of an appropriate discretization of the space. Given $\mathcal{A} \subset$ $\mathcal{P}(X), x \in X$, and $K \subset X$, let $|\mathcal{A}|:=\bigcup\{A \mid A \in \mathcal{A}\}, \mathcal{A}(x):=\{A \in \mathcal{A} \mid x \in A\}$ and $\mathcal{A}(K):=\{A \in \mathcal{A} \mid A \cap K \neq \emptyset\}$. A grid in $X$ is a family of sets $\mathcal{G} \subset \mathcal{P}(X)$ such that any element of $\mathcal{G}$ is equal to the closure of its interior, any two different elements of $\mathcal{G}$ have disjoint interiors and for any compact set $K \subset X$ the family $\mathcal{G}(K)$ is non-empty and finite. A subfamily of a grid is called a subgrid. Let $\gamma>0$. 
The standard $\gamma$-grid in $\mathbb{R}^{d}$ is the collection

$$
\mathcal{G}_{\gamma}^{d}:=\mathcal{G}_{\gamma}:=\left\{\left[n_{1} \gamma,\left(n_{1}+1\right) \gamma\right] \times \cdots \times\left[n_{d} \gamma,\left(n_{d}+1\right) \gamma\right] \mid\left(n_{1}, \ldots n_{d}\right) \in \mathbb{Z}^{d}\right\} .
$$

The elements of a given grid will serve as quants of space and sets will be represented by finite families of such sets. In the quantized space the concept of a minimal neighborhood makes sense and can be formalized as follows. If $\mathcal{G}$ is a grid and $\mathcal{A} \subset \mathcal{G}$, then $\mathcal{A}_{\mathcal{G}}^{*}:=\mathcal{A}^{*}:=\{G \in \mathcal{G} \mid \exists A \in \mathcal{A}$ such that $G \cap A \neq \emptyset\}$.

A compact set $K \subset X$ is representable over a grid $\mathcal{G}$ if $K$ is a finite union of intersections of some subgrids of $\mathcal{G}$. $K$ is strongly representable over $\mathcal{G}$ if there exists a grid $\mathcal{G}_{0} \subset \mathcal{G}$ such that $K=\left|\mathcal{G}_{0}\right|$. Since such a $\mathcal{G}_{0}$ is then unique, we will denote it by $\mathcal{G}(K)$.

Assume $\mathcal{G}$ and $\mathcal{G}^{\prime}$ are grids in $X$. A multivalued map $F: X \rightarrow \mathcal{P}(X)$ is representable over the pair $\left(\mathcal{G}, \mathcal{G}^{\prime}\right)$ if $\operatorname{dom} F$ is a representable set over $\mathcal{G}$, for every $x \in \operatorname{dom} F$ the set $F(x)$ is representable over $\mathcal{G}^{\prime}$ and $\mathcal{A}(x)=\mathcal{A}(y)$ implies $F(x)=F(y)$. Let $U \subset X$ and let $f: U \rightarrow X$ be a single valued map. A multivalued map $F: X \rightarrow \mathcal{P}(X)$ is a representation of $f$ over the pair $\left(\mathcal{G}, \mathcal{G}^{\prime}\right)$ if $F$ is representable over $\left(\mathcal{G}, \mathcal{G}^{\prime}\right)$ and $f$ is a selector of $F_{\mid U}$, i.e. $U \subset \operatorname{dom} F$ and for every $x \in U$ we have $f(x) \in F(x)$.

In order to obtain appropriate multivalued representations of single valued maps we will use the concept of a combinatorial multivalued map i.e., a map $\mathcal{F}: \mathcal{G} \rightarrow$ $\mathcal{P}\left(\mathcal{G}^{\prime}\right)$ such that $\operatorname{dom} \mathcal{F}$ is finite and for any $G \in \operatorname{dom} \mathcal{F}$ the family $\mathcal{F}(G)$ is finite. The map $\mathcal{F}$ will be called a representation of $f$ over the pair $\left(\mathcal{G}, \mathcal{G}^{\prime}\right)$ if $U \subset|\operatorname{dom} \mathcal{F}|$ and for every $G \in \operatorname{dom} \mathcal{F}$ and $x \in U \cap G$ we have $f(x) \in|\mathcal{F}(G)|$. The diameter of $\mathcal{F}$ $($ denoted diam $\mathcal{F})$ is the maximum of diameters of $|\mathcal{F}(x)|$ over all $x \in \operatorname{dom} \mathcal{F}$. With a combinatorial multivalued map $\mathcal{F}$ we associate the following two multivalued maps

$$
\begin{aligned}
& \lfloor\mathcal{F}\rfloor:|\mathcal{A}| \ni x \rightarrow \bigcap\{|\mathcal{F}(A)| \mid A \in \mathcal{A}(x)\} \in \mathcal{P}\left(\left|\mathcal{A}^{\prime}\right|\right), \\
& \lceil\mathcal{F}\rceil:|\mathcal{A}| \ni x \rightarrow \bigcup\{|\mathcal{F}(A)| \mid A \in \mathcal{A}(x)\} \in \mathcal{P}\left(\left|\mathcal{A}^{\prime}\right|\right) .
\end{aligned}
$$

One can easily prove the following theorem (cf. [18]).

Theorem 4.1. The map $\lfloor\mathcal{F}\rfloor$ is lsc, the map $\lceil\mathcal{F}\rceil$ is usc and both maps are representable. Moreover, if $\mathcal{F}$ is a representation of $f: U \rightarrow X$, then $\lfloor\mathcal{F}\rfloor$ and $\lceil\mathcal{F}\rceil$ are, also, representations of $f$.

Now we are ready to start discretizing the assumptions of Theorem 2.1. Let $I$ be an interval in $\mathbb{Z}$ with $0 \in I$. A single valued mapping $\sigma: I \rightarrow X$ is a solution for $F$ through $x \in X$ if $\sigma(n+1) \in F(\sigma(n))$ for all $n, n+1 \in I$, and $\sigma(0)=x$. Let Inv $N$ denote the set of $x \in N$ such that there exists a solution $\sigma: \mathbb{Z} \rightarrow N$ for $F$ through $x$. The following concepts were introduced in [7]. A compact subset $N \subset X$ is called an isolating neighborhood for $F$ if $B_{\operatorname{diam}_{N} F}(\operatorname{Inv} N) \subset \operatorname{int} N$ and an isolating block for $F$ if $B_{\operatorname{diam}_{N} F}\left(F^{*-1}(N) \cap N \cap F(N)\right) \subset \operatorname{int} N$. It is straightforward to verify that every isolating block is an isolating neighborhood.

Let $N$ be an isolating neighborhood for $F$. A pair $P=\left(P_{1}, P_{2}\right)$ of compact subsets $P_{2} \subset P_{1} \subset N$ is called an index pair if $F\left(P_{i}\right) \cap N \subset P_{i}$ for $i=1,2$, $F\left(P_{1} \backslash P_{2}\right) \subset N$ and Inv $N \subset \operatorname{int}\left(P_{1} \backslash P_{2}\right)$. It turns out that under certain conditions called admissibility, multivalued maps induce maps in cohomology (see [7],[18] — for purposes of this paper it is enough to know that lsc or usc convex-valued maps are admissible). Thus, as in Section 3, one can define the index map and the Conley index $\operatorname{Con}(N, F)$. 


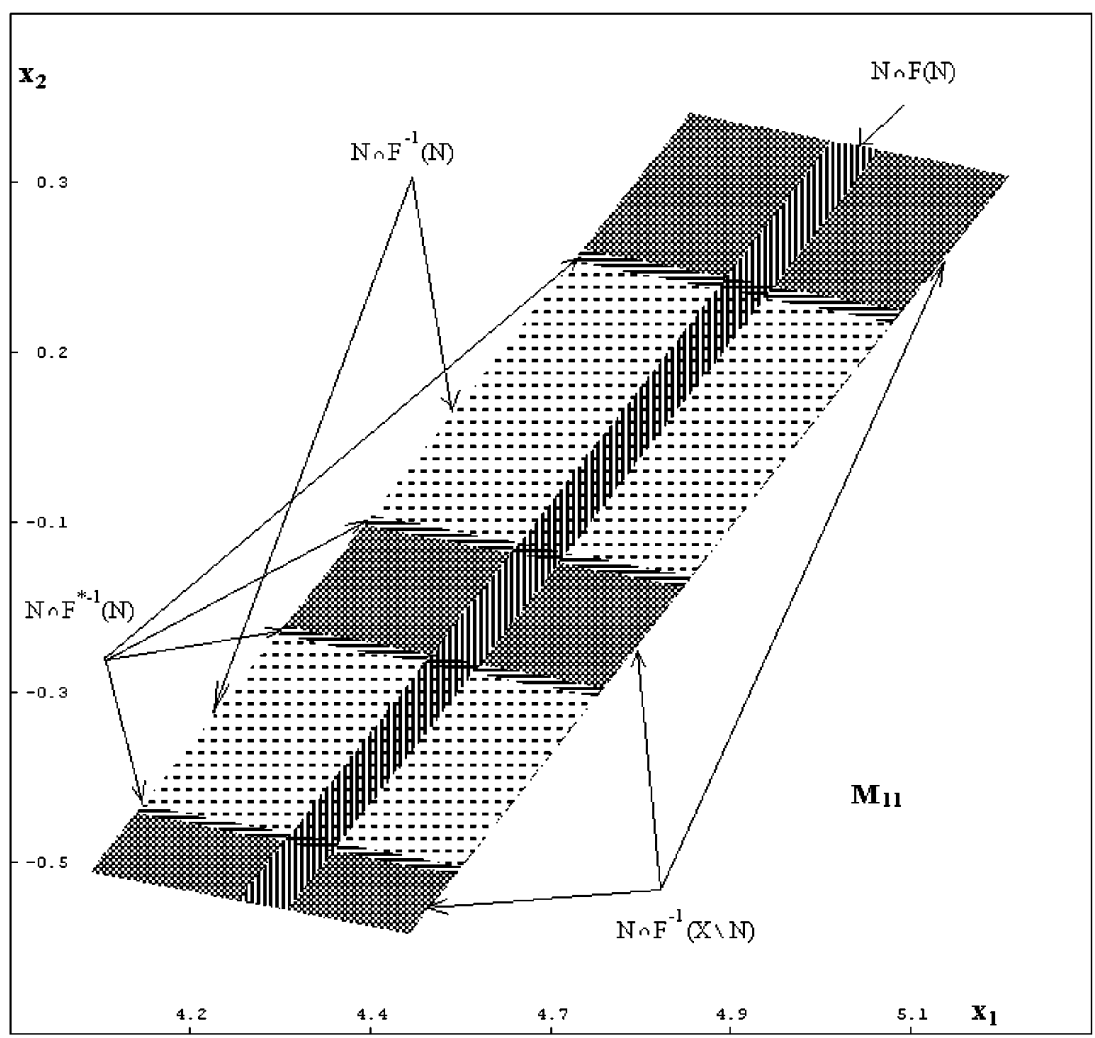

Figure 3. Behavior of $g$ on $M_{11}$

Theorem 4.2 (see [7], [18]). Assume $F$ is an admissible representation of $f, N$ is an isolating neighborhood for $F$ and $P=\left(P_{1}, P_{2}\right)$ is an index pair for $F$ in $N$. Then $N$ is also an isolating neighborhood for $f$ and $P$ is an index pair for $f$ in $N$. Furthermore, if $F$ is admissible, then $\operatorname{Con}(N, F)=\operatorname{Con}(N, f)$.

The above theorem indicates that for the verification of the assumptions of Theorem 2.1 it is sufficient to find a multivalued representation of the Poincaré map for which $N_{i j}$ are isolating neighborhoods and find the respective Conley indexes. Ideally one makes the $N_{i j}$ isolating blocks, because then, as the following theorem shows, finding the necessary Conley indexes is simple.

Theorem 4.3 (see [18]). Assume $\mathcal{G}$ is a grid in $X$ and $\mathcal{F}: \mathcal{G} \rightarrow \mathcal{P}(\mathcal{G})$ is a combinatorial multivalued map. If $\mathcal{N}=\operatorname{dom} \mathcal{F}$ is such that

$$
\operatorname{dist}_{\text {sup }}\left(\left|\mathcal{F}^{*-1}(\mathcal{N}) \cap \mathcal{F}(\mathcal{N})^{*}\right|, \text { bd }|\mathcal{N}|\right)>\operatorname{diam} \mathcal{F},
$$

then $|\mathcal{N}|$ is an isolating block for $\lfloor\mathcal{F}\rfloor$ and $\left(|\mathcal{N}|,\left|\mathcal{N} \backslash \mathcal{F}^{-1}(\mathcal{N})\right|\right)$ is an index pair for $\lfloor\mathcal{F}\rfloor$ in $|\mathcal{N}|$.

Figure 3 represents an enlargement of the parallelogram $M_{11}$. Actually the sets $M_{i j}, i, j \in\{0,1\}$ are not exactly parallelograms but coverings of parallelograms by elements of a standard grid in $\mathbb{R}^{2}$. However the elements of the grid are too small to be shown. Various shadings in Figure 3 indicate the behavior of a combinatorial multivalued representation of the Poincaré map $g$. The pictures obtained 
for $M_{00}, M_{01}, M_{10}$ look practically the same. Figure 3 explains how the index pair in Figure 2 was obtained.

Note that a theorem analogous to Theorem 4.3 may also be proved for $\lceil\mathcal{F}\rceil$ (see [18]). The advantage of $\lfloor\mathcal{F}\rfloor$ over $\lceil\mathcal{F}\rceil$ lies in the fact that if $\mathcal{F}$ is a representation of a single valued map $f$, then $\operatorname{diam}\lfloor\mathcal{F}\rfloor \leq \operatorname{diam} \mathcal{F}$ but $\operatorname{diam}\lceil\mathcal{F}\rceil \leq 2 \operatorname{diam} \mathcal{F}$. Thus one expects that verifying if a given set is an isolating block may be more expensive in the case of $\lceil\mathcal{F}\rceil$.

\section{PoINCARÉ MAPS}

As we mentioned in the introduction the successful verification of the assumptions of Theorem 2.1 requires constructing a representation of the Poincaré map of relatively small size. This may be a problem, because error bounds grow exponentially with time. In order to avoid lengthy integration, some intermediate sections were introduced, multivalued representations were computed for the intermediate Poincaré maps and the required representation of the full Poincaré map was taken as the composition.

We will explain now the theoretical background of the intermediate sections approach.

Let $Z \subset X$ be compact. For $x \in Z$ define $\omega_{Z}(x):=\sup \left\{t \in \mathbb{R}^{+} \mid \varphi([0, t], x) \subset\right.$ $Z$ \}. The set $\Xi \subset X$ is a local section in $Z$ if for every $x \in Z$ there is

$$
\operatorname{card}\left\{t \in\left[0, \omega_{Z}(x)\right] \mid \varphi(t, x) \in \Xi\right\} \leq 1 .
$$

To define a Poincaré map with respect to $\Xi$ one needs to know how points cross the section. To do this, given a $\Theta \subset \Xi$, define a partial map on $X$ by $T_{\Theta, 0}(x):=$ $\sup \left\{s \in \mathbb{R}^{+} \mid \varphi([0, s), x) \cap \Theta=\emptyset\right\}$. Observe that $T_{\Theta, 0}(x)$ represents the first time that the forward orbit of $x$ intersects the section $\Xi$ in $\Theta$. The actual point of crossing is returned by another partial map given by $\operatorname{dom} \Psi_{\Theta, 0}:=\operatorname{dom} T_{\Theta, 0}$ and $\Psi_{\Theta, 0}(x)=\varphi\left(T_{\Theta, 0}(x), x\right)$ for $x \in \operatorname{dom} T_{\Theta, 0}$. Denote $E(Z, \Xi):=\left\{x \in Z \cap \operatorname{dom} \Psi_{\Xi, 0} \mid\right.$ $\left.\varphi\left(\left[0, T_{\Xi, 0}(x)\right], x\right) \subset Z\right\}$. Assume $A \subset X$ is compact and put $\tilde{\varphi}_{\Xi}(A):=\{y \mid \exists x \in$ $A \cap \operatorname{dom} T_{\Xi, 0}$ such that $\left.y \in \varphi\left(\left[0, T_{\Xi, 0}(x)\right], x\right)\right\}$. We will say that $A$ precedes $\Xi$ if there exists a compact set $W \subset X$ such that $\Xi$ is a local section in $W, A \subset$ $E(W, \Xi)$ and there exists a $T>0$ such that $T_{\Xi, 0}(x) \in(0, T)$ for $x \in A$.

Lemma 5.1. If A precedes $\Xi$, then $\left.T_{\Xi, 0}\right|_{A}$ and $\left.\Psi_{\Xi, 0}\right|_{A}$ are continuous.

Proof. Let $\left\{x_{n}\right\} \subset A$ be such that $x_{n} \rightarrow x \in A$ and $t_{n}:=T_{\Xi, 0}\left(x_{n}\right) \rightarrow t \in[0, \infty]$. Choose $W \subset X$ such that $\Xi$ is a local section in $W, A \subset E(W, \Xi)$ and there exists a $T>0$ such that $T_{\Xi, 0}(x) \in(0, T)$ for $x \in A$. It follows that $t<\infty$. Since $\Xi$ is closed, we get $\varphi(t, x) \in \Xi$, and consequently $T_{\Xi, 0}(x) \leq t$. It cannot be that $T_{\Xi, 0}(x)<t$, because $\varphi([0, t], x) \subset W$ and $\Xi$ is a local section in $W$. Thus $t=T_{\Xi, 0}(x)$, which shows that $\left.T_{\Xi, 0}\right|_{A}$ is continuous. The continuity of $\left.\Psi_{\Xi, 0}\right|_{A}$ follows immediately from its definition.

Observe that given $x \in \Theta \subset \Xi, T_{\Theta, 0}(x)=0$. Thus, in order to obtain a first return map we define $T_{\Theta}(x):=\sup \left\{s \in \mathbb{R}^{+} \mid \operatorname{card}\{\varphi([0, s), x) \cap \Theta\}=1\right\}$. In the sequel we are interested in the partial map $\Psi_{\Theta}$ given by $\operatorname{dom} \Psi_{\Theta}=\operatorname{dom} T_{\Theta}$ and $\Psi_{\Theta}(x):=\varphi\left(T_{\Theta(x)}, x\right)$ for $x \in \operatorname{dom} T_{\Theta}$. We will call it the Poincaré map associated with $\Theta$. The following lemma shows how the Poincaré map can be glued together from small pieces. 
Lemma 5.2. Assume $N \subset \Theta \subset \Xi$ and $\Xi_{1}, \ldots, \Xi_{n}=\Xi, Z_{1}, \ldots, Z_{n}$ for some $n \geq 1$ are compact subsets of $X$ such that $\Xi$ is a local section in $Z_{1}$ and $Z_{i} \cap \Theta=\emptyset$ for $i=2,3, \ldots, n-1$. If $A_{i} \subset X$ for $i=0,1,2, \ldots, n$ are compact sets such that $N \subset A_{0}, Z_{n} \cap \Xi \subset \Theta, \Psi_{\Xi_{i}, 0}\left(A_{i-1}\right) \subset A_{i}$, for $i=1,2, \ldots, n$ and

$$
A_{i-1} \text { precedes } \Xi_{i} \text { and } \tilde{\varphi}_{\Xi_{i}}\left(A_{i-1}\right) \subset Z_{i} \text { for } i=1,2, \ldots, n,
$$

then

$$
\begin{gathered}
\left.\Psi_{\Xi_{i}, 0}\right|_{A_{i-1}} \text { are continuous for } i=1,2, \ldots, n, \\
\quad N \subset \operatorname{dom} \Psi_{\Theta}, \\
\left.\Psi_{\Theta}\right|_{N}=\left(\left.\left.\Psi_{\Xi_{i}, 0}\right|_{A_{i-1}} \circ \cdots \circ \Psi_{\Xi_{i}, 0}\right|_{A_{0}}\right)_{\mid N},
\end{gathered}
$$

and $\left.\Psi_{\Theta}\right|_{N}$ is continuous.

Proof. Property (7) follows immediately from Lemma 5.1. To prove (8) take $x \in N$. Put $t_{0}:=0, x_{0}:=x$ and for $i \leq n$ define recursively $t_{i}:=t_{i-1}+T_{\Xi_{i}, 0}\left(x_{i-1}\right), x_{i}:=$ $\varphi\left(T_{\Xi_{i}, 0}\left(x_{i-1}\right), x_{i-1}\right)$. Then $0=t_{0}<t_{1}<\ldots<t_{n}$ and $\varphi\left(t_{i}, x\right) \in Z_{i} \cap A_{i}$ for $i=0,1, \ldots, n$. In particular $\varphi\left(t_{n}, x\right) \in Z_{n} \cap \Xi \subset \Theta$. This shows (8). To prove (9), first observe that the domains of maps on both sides of (9) are equal and the right-hand side evaluated at $x \in N$ is $\varphi\left(t_{n}, x\right)$. To show that this is also the lefthand side at $x$ it is sufficient to prove that $\varphi(t, x) \notin \Theta$ for $0<t<t_{n}$. For this end select $i \in\{1,2, \ldots, n\}$ such that $t_{i-1}<t \leq t_{i}$. If $i \in\{2, \ldots, n-1\}$, then by (6) $\varphi(t, x)=\varphi\left(t-t_{i-1}, x_{i-1}\right) \in \tilde{\varphi}_{\Xi_{i}}\left(A_{i-1}\right) \subset Z_{i}$ and consequently $\varphi(t, x) \notin \Theta$. If $0<t \leq t_{1}$, then $\varphi(t, x) \notin \Theta$, because $x=\varphi(0, x) \in N \subset \Theta \subset \Xi, \varphi([0, t], x) \subset Z_{1}$ and $\Xi$ is a local section in $Z_{1}$. Similarly, if $t_{n-1}<t<t_{n}$, then $\varphi(t, x) \notin \Xi$ and in particular $\varphi(t, x) \notin \Theta$, because $\varphi\left(t_{n}, x\right) \in \Xi, \varphi\left(\left[t, t_{n}\right], x\right) \subset Z_{n}$ and $\Xi=\Xi_{n}$ is a local section in $Z_{n}$. Finally the continuity of $\left.\Psi_{\Theta}\right|_{N}$ follows immediately from (7) and (9).

\section{Representations of Poincaré maps in $\mathbb{R}^{d}$}

Let $\varphi: \mathbb{R} \times \mathbb{R}^{d} \rightarrow \mathbb{R}^{d}$ denote the flow generated by (4). Let $Z \subset \mathbb{R}^{d}$ be a compact subset. For $s>0$ denote $Z_{s}:=\{x \in Z \mid \varphi([0, s], x) \subset Z\}$.

The main tool in obtaining multivalued representations of Poincaré maps are multivalued representations of $t$-translation operators along the flow. The first step in constructing such representations is a way of rigorously enclosing the $t$-translation operator at a given argument. This may be obtained via the following theorem.

Theorem 6.1 (see [19]). Assume the flow $\varphi$ is generated by (4), where $V$ is a polynomial vector field. Let $\Phi: \mathbb{R} \times \mathbb{R}^{d} \rightarrow \mathbb{R}^{d}$ be an explicit Runge-Kutta method for (4) (see [4]) and let $\langle\Phi\rangle$ denote its machine evaluation (see [19]). Then for every $h>0$ and $x \in Z_{h}$

$$
\|\varphi(h, x)-\langle\Phi\rangle(h, x)\|_{\text {sup }} \leq E_{Z}^{\Phi}(h),
$$

where $E_{Z}^{\Phi}(h)$ is a polynomial depending on $\Psi$ and explicitly given in [19].

The formula for the polynomial $E_{Z}(h)$ is lengthy so we omit it here. The interested reader may consult [19]. Finding the function $E_{Z}$ from these formulas is an elementary task but, typically arduous or even practically unrealizable without the use of a computer. Even if $V$ is a polynomial of degree 2, as in the case of Lorenz equations, the computation of $E_{Z}$ involves polynomials of degree 14 . We used Mathematica to find the formula for $E_{Z}$ for the Lorenz equations. 
Theorem 6.1 allows one to find an enclosure of the $t$-translation operator at a given point. In order to be able to construct a multivalued representation of the $t$-translation operator, we need to extend the estimates given by Theorem 6.1 to nearby points. For this end let us introduce the following definitions.

If $A$ is a matrix $\left(a_{i j}\right)_{i, j=1, \ldots, d}$, then $\sigma(A)$ will stand for the logarithmic norm of $A$ (see [4], Definition 10.4). In particular, in case of the supremum norm $\sigma_{\text {sup }}(A)=\max \left\{a_{i i}+\sum_{j \neq i}\left|a_{i j}\right|\right\}$, (see [4], Theorem 10.5). Let $D V(x)$ denote the linearization of $V$ at $x \in \mathbb{R}^{d}$ and let $L_{\sigma} \in \mathbb{R}^{+}$be a constant such that $|\sigma(D V(x))-\sigma(D V(y))| \leq L_{\sigma}\|x-y\|$ for $x, y \in \mathbb{R}^{d}$. Note that in case of the Lorenz equations and the supremum norm one can take $L_{\sigma}=2$. Let $Z \subset \mathbb{R}^{d}$ be a fixed compact subset. In the sequel we assume that $M_{Z}, L_{Z} \in \mathbb{R}^{+}$are chosen so that $M_{Z} \geq \sup \{\|V(x)\|: x \in Z\}$ and $L_{Z} \geq \sup \{\|D V(x)\|: x \in Z\}$. Let $\Phi=\Phi_{V}: \mathbb{R}^{d} \times \mathbb{R} \rightarrow \mathbb{R}^{d}, E=E_{Z}: \mathbb{R}^{+} \rightarrow \mathbb{R}^{+}$denote the Runge-Kutta method and the error estimate as in Theorem 6.1.

Lemma 6.2. If $r>0, s>0$ and $x \in Z$ are such that $\operatorname{dist}(x, \operatorname{bd} Z)>M_{Z} s+r$, then

$$
\varphi([0, s], B(x, r)) \subset Z
$$

Moreover, if $u<1$, then

$$
\varphi(s, B(x, r)) \subset B\left(\langle\Phi\rangle(s, x), r\left(1+u+u^{2}\right)+E_{Z}(s)\right),
$$

where $u:=s\left(L_{\sigma}\left(M_{Z} s+r\right)+\sigma(D V(x))\right)$.

Proof. To show (10) take $y \in B(x, r)$ and let $s_{0}:=\sup \{t \in[0, s] \mid \varphi([0, t], y) \subset Z\}$. Then $s_{0} \leq s$ and

$$
\|\varphi(t, y)-x\| \leq\|\varphi(t, y)-y\|+\|y-x\| \leq M_{Z} s+r
$$

for $t \in\left[0, s_{0}\right]$ and $y \in B(x, r)$. It needs to be shown that $s_{0}=s$. If $s_{0}<s$, then $\varphi\left(s_{0}, y\right) \in \operatorname{bd} Z$ and we get from (12) $\operatorname{dist}(x, \operatorname{bd} Z) \leq\left\|\varphi\left(s_{0}, y\right)-x\right\| \leq M_{Z} s+r$, a contradiction. This proves (10).

Now define $D_{\text {loc }}(x, r, s):=\bigcup\{\varphi([0, s], y) \mid y \in B(x, r)\}$. It follows from (12) that $D_{\text {loc }}(x, r, s) \subset B\left(x, M_{Z} s+r\right)$. For $z \in B\left(x, M_{Z} s+r\right)$ we have

$$
\begin{aligned}
& \sigma(D V(z)) \leq|\sigma(D V(z))-\sigma(D V(x))|+\sigma(D V(x)) \\
& \quad \leq L_{\sigma}|| x-z \|+\sigma(D V(x)) \leq L_{\sigma}\left(M_{Z} s+r\right)+\sigma(D V(x)) .
\end{aligned}
$$

To prove (11) take $y \in B(x, r)$. Then from ([4], Theorem 10.6)

$$
\begin{aligned}
\|\varphi(s, y)-\Phi(s, x)\| & \leq\|\varphi(s, y)-\varphi(s, x)\|+\|\varphi(s, x)-\Phi(s, x)\| \\
& \leq r e^{s\left(L_{\sigma}\left(M_{Z} s+r\right)+\sigma(D V(x))\right)}+E_{Z}(s)=r e^{u}+E_{Z}(s) .
\end{aligned}
$$

Property (11) follows now from the fact that for $0 \leq u \leq 1$ the estimate $e^{u}<$ $1+u+u^{2}$ is satisfied.

For $a, b \in \mathbb{R}^{p}$ let $\overline{a b}:=\{\lambda a+(1-\lambda) b \mid \lambda \in[0,1]\}$ denote the segment in $\mathbb{R}^{p}$ joining $a, b$. The following lemma will let us find a representation of the Poincaré map.

Lemma 6.3. Assume $h>0, a, b \in \mathbb{R}^{p}$ and $\alpha, \beta>0$ are such that $x \in Z_{h} \cap B(a, \alpha)$ implies $\varphi(h, x) \in B(b, \beta)$. Then, for any $t \in[0, h]$ and $x \in B(a, \alpha)$ we have $\varphi(t, x) \in B\left(\overline{a b}, \max (\alpha, \beta)+L_{Z} M_{Z} h^{2}\right)$. 
Proof. We have

$$
\begin{aligned}
\operatorname{dist}(\varphi(t, x), \overline{a b}) \leq & \left\|\varphi(t, x)-\left(a+\frac{t}{h}(b-a)\right)\right\| \leq\|\varphi(t, x)-(x+V(x) t)\| \\
& +\left\|(x+V(x) t)-\left(x+\frac{t}{h}(\varphi(h, x)-x)\right)\right\| \\
& +\left\|x+\frac{t}{h}(\varphi(h, x)-x)-\left(a+\frac{t}{h}(b-a)\right)\right\| .
\end{aligned}
$$

Using a Taylor expansion we get for some $\theta, \theta_{1} \in[0, h]$

$$
\begin{aligned}
\operatorname{dist}(\varphi(t, x), \overline{a b}) \leq \frac{1}{2}\left\|\frac{\partial^{2}}{\partial t^{2}} \varphi(\theta t, x)\right\| t^{2}+\left\|\frac{\partial^{2}}{\partial t^{2}} \varphi\left(\theta_{1} h, x\right) \frac{h}{2}\right\| t \\
\quad+\left\|x\left(1-\frac{t}{h}\right)+\frac{t}{h}(\varphi(h, x))-\left[a\left(1-\frac{t}{h}\right)+\frac{t}{h} b\right]\right\| .
\end{aligned}
$$

Now, since $x \in Z_{h}$, the derivative $\frac{\partial^{2}}{\partial t^{2}} \varphi$ may be estimated by $L_{Z} M_{Z}$, i.e.

$$
\begin{aligned}
\operatorname{dist}(\varphi(t, x), \overline{a b}) & \leq \frac{L_{Z} M_{Z} t^{2}}{2}+\left\|\frac{\partial^{2}}{\partial t^{2}} \varphi\left(\theta_{1} h, x\right)\right\| \frac{t h}{2} \\
& +\|x-a\|\left(1-\frac{t}{h}\right)+\|\varphi(h, x)-b\| \frac{t}{h} \leq L_{Z} M_{Z} h^{2}+\max (\alpha, \beta) .
\end{aligned}
$$

The above lemma implies that if a point $\varphi(x, t)$ on the trajectory of $x$ is contained in a ball $B(a, \alpha)$ which lies entirely on one side of a surface and $\varphi(t+h, x)$ lies in a ball $B(b, \beta)$ entirely on the other side of the surface, then the trajectory of $x$ intersects the surface and the point of intersection lies in the ball $B\left(\overline{a b}, \max (\alpha, \beta)+L_{Z} M_{Z} h^{2}\right)$. In order to be sure that the point of intersection is actually the value of the Poincaré map we need some way of guaranteeing that this is the first point of intersection. We will need another lemma for this purpose. Let $\Xi$ be an oriented affine hyperplane in $\mathbb{R}^{d}$ given by $\Xi:=\left\{x \in \mathbb{R}^{d} \mid x \cdot v_{\Xi}=c_{\Xi}\right\}$, where $v_{\Xi} \in \mathbb{R}^{d}$ is a vector orthogonal to $\Xi$ and indicating its orientation and $c_{\Xi} \in \mathbb{R}$ is a constant.

Lemma 6.4. If $V(x) \cdot v_{\Xi} \neq 0$ for $x \in W$, then $\Xi$ is a local section in $W$.

Proof. Assume the contrary. Then there exists a point $x \in W$ and a $t>0$ such that $\varphi([0, t], x) \subset W$ and $x, \varphi(t, x) \in \Xi$. Consider the function $\tau:[0, t] \ni s \rightarrow$ $(\varphi(s, x)) \cdot v_{\Xi}-c_{\Xi} \in \mathbb{R}$. Since $\tau(0)=\tau(t)=0$, there exists an $s \in[0, t]$ such that $0=\tau^{\prime}(s)=V(\varphi(s, x)) \cdot v_{\Xi}$, a contradiction.

Although the above lemmas are valid for any norm, the supremum norm is easiest to implement in our algorithms. In order to make use of Lemma 6.3 we need formulas, which we shall now present, for the distance in the supremum norm from a point to a line and a plane.

For $x=\left(x_{1}, x_{2}, \ldots, x_{d}\right) \in \mathbb{R}^{d}$ let $\operatorname{Sgn} x:=\left(\operatorname{sgn} x_{1}, \operatorname{sgn} x_{2}, \ldots, \operatorname{sgn} x_{d}\right)$. We will say that an $x \in \mathbb{R}^{d}$ lies above (below) the hyperplane $\Xi$ if $x \cdot v_{\Xi}>c_{\Xi}\left(x \cdot v_{\Xi}<c_{\Xi}\right)$. We define the oriented supremum norm distance from $x \in \mathbb{R}^{d}$ to $\Xi$ by

$$
\text { ordist }_{\text {sup }}(x, \Xi)= \begin{cases}\operatorname{dist}_{\text {sup }}(x, \Xi), & x \text { lies above } \Xi, \\ -\operatorname{dist}_{\text {sup }}(x, \Xi), & x \text { lies below } \Xi, \\ 0, & \text { otherwise. }\end{cases}
$$


Lemma 6.5. The oriented supremum norm distance and the supremum norm distance from the point $x \in \mathbb{R}^{d}$ to the hyperplane $\Xi$ are given by

$$
\operatorname{ordist}_{\text {sup }}(x, \Xi)=\frac{x \cdot v_{\Xi}-c_{\Xi}}{\left\|v_{\Xi}\right\|_{1}}, \quad \operatorname{dist}_{\text {sup }}(x, \Xi)=\frac{\left|x \cdot v_{\Xi}-c_{\Xi}\right|}{\left\|v_{\Xi}\right\|_{1}} .
$$

Proof. Obviously it is sufficient to prove only the right equation in (13). Let $\bar{x} \in \Xi$ be such that $\operatorname{dist}_{\text {sup }}(x, \Xi)=\|\bar{x}-x\|_{\text {sup }}$. It is easy to see that $\bar{x}$ may be chosen so that $\bar{x}-x=a \operatorname{Sgn}\left(v_{\Xi}\right)$ for some $a \in \mathbb{R}$. Since $\bar{x} \in \Xi$ we have $\left(x+a \operatorname{Sgn} v_{\Xi}\right) \cdot v_{\Xi}-c_{\Xi}=0$. It follows that $x \cdot v_{\Xi}-c_{\Xi}=-a\left\|v_{\Xi}\right\|_{1}$ and $\|\bar{x}-x\|_{\text {sup }}=|a||| \operatorname{Sgn} v_{\Xi} \|_{\text {sup }}=|a|=$ $\frac{\left|x \cdot v_{\Xi}-c \Xi\right|}{\left\|v_{\Xi}\right\|_{1}}$.

Let $l$ be a line in $\mathbb{R}^{3}$ given by $l:=\left\{p_{l}+t v_{l} \mid t \in \mathbb{R}\right\}$, where $p_{l}, v_{l} \in \mathbb{R}^{3}$.

Lemma 6.6. The supremum norm distance from the point $x \in \mathbb{R}^{3}$ to the line $l$ is given by

$$
\operatorname{dist}_{\text {sup }}(x, l)=\max \left\{\operatorname{dist}_{\text {sup }}(\pi(x), \pi(l)) \mid \pi \in \operatorname{Proj}_{3}\right\},
$$

where Proj $j_{3}$ denotes the set of the three canonical projections of $\mathbb{R}^{3}$ onto $\mathbb{R}^{2}$.

Proof. Since every canonical projection is 1-Lipschitz in the supremum metric, the left-hand side of (14) is obviously greater or equal the right-hand side. To prove the other inequality select $\bar{x} \in l$ to be such that $\operatorname{dist}_{\text {sup }}(x, l)=\|\bar{x}-x\| \|_{\text {sup }}$ and put $w:=\left(w_{1}, w_{2}, w_{3}\right):=\bar{x}-x$. It is easy to observe that $\bar{x}$ may be chosen so that among the numbers $\left\{\left|w_{1}\right|,\left|w_{2}\right|,\left|w_{3}\right|\right\}$ at least two are equal and the third is not greater than the other two. This means that among the three canonical projections we can select one, denoted by $\pi$, such that $\operatorname{dist}_{\text {sup }}(\pi(x), \pi(l))=\|\pi(\bar{x})-\pi(x)\|_{\text {sup }}$. It follows that

$$
\begin{gathered}
\operatorname{dist}_{\text {sup }}(x, l)=\|w\|_{\text {sup }}=\|\pi(w)\|_{\text {sup }}=\|\pi(\bar{x}-x)\|_{\text {sup }}=\operatorname{dist}_{\text {sup }}(\pi(x), \pi(l)) \\
\leq \max \left\{\operatorname{dist}_{\text {sup }}(\pi(x), \pi(l)) \mid \pi \in \operatorname{Proj}_{3}\right\} .
\end{gathered}
$$

\section{INTERVAL ARITHMETIC AND ŁUKASIEWICZ LOGIC}

In this section we briefly recall the main ideas concerning the interval arithmetic and Eukasiewicz logic. The set of intervals over $A \subset \overline{\mathbb{R}}$ is defined as $\mathcal{I}(A):=$ $\left\{\left[a^{-}, a^{+}\right] \mid a^{-}, a^{+} \in A, a^{-} \leq a^{+}\right\}$. The set of real numbers may be viewed as a subset of $\mathcal{I}(\overline{\mathbb{R}})$ via the natural embedding $\overline{\mathbb{R}} \ni x \rightarrow[x, x] \in \mathcal{I}(\overline{\mathbb{R}})$. In the opposite direction, one has the two functions xlower : $\mathcal{I}(\overline{\mathbb{R}}) \ni\left[a^{-}, a^{+}\right] \rightarrow a^{-} \in \overline{\mathbb{R}}$ and upper : $\mathcal{I}(\overline{\mathbb{R}}) \ni\left[a^{-}, a^{+}\right] \rightarrow a^{+} \in \overline{\mathbb{R}}$ which select the lower and upper bounds of the interval. In the sequel we will treat the minimum and maximum of two numbers $x, y \in \overline{\mathbb{R}}$ as a binary operation on $\overline{\mathbb{R}}$ denoted by $x \wedge y:=\min (x, y)$ and $x \vee y:=\max (x, y)$ respectively. Let $\diamond \in\{+,-, *, /, \wedge, \vee\}$. The arithmetic operations are extended to $I, J \in \mathcal{I}(\overline{\mathbb{R}})$ by $I \diamond J:=\{a \diamond b \mid a \in I, b \in J\}$. Let $\hat{\mathbb{R}} \subset \overline{\mathbb{R}}$ be a finite set of real numbers satisfying the 64-bit IEEE standards. We will call this set the set of representable numbers. Intervals over the set of representable numbers are called representable intervals. If $I \in \mathcal{I}(\overline{\mathbb{R}})$, then the smallest representable interval containing $I$ will be called the representation of $I$ and denoted by $\uparrow(I)$. One then defines the machine arithmetic operations on representable intervals $I, J \in \mathcal{I}(\hat{\mathbb{R}})$ by 
$I\langle\diamond\rangle J:=\uparrow(I \diamond J)$. Note that $\uparrow(I \diamond J)$ may be easily found from the endpoints of $I, J$.

If $Q: \mathbb{R}^{d} \rightarrow \mathbb{R}$ is a rational function, then replacing the standard arithmetic operations by the corresponding machine operations on intervals one obtains a function $\mathcal{Q}: \mathcal{I}(\hat{\mathbb{R}})^{d} \rightarrow \mathcal{I}(\hat{\mathbb{R}})$ (note that such a function need not be unique because the machine arithmetic operations on intervals are not associative). We will call such a function $\mathcal{Q}$ an interval representation of $Q$. The usefulness of intervals lies in the following proposition which one can easily prove by induction on the complexity of $Q$.

Proposition 7.1. If $Q\left(x_{1}, \ldots, x_{n}\right)$ is a rational function and $I_{1}, \ldots, I_{n} \in \mathcal{I}(\hat{\mathbb{R}})$ are intervals such that $x_{i} \in I_{i}$, then $Q\left(x_{1}, \ldots, x_{n}\right) \in \mathcal{Q}\left(I_{1}, \ldots, I_{n}\right)$.

It is essential to be able to guarantee that whatever is proved for intervals is also true for any selection of numbers in the intervals. To make it work we need to extend the two classical logical values: 0 (false) and 1 (true). Following Łukasiewicz [9], we add the third value $\frac{1}{2}$ and we define the basic logical operations (conjunction, alternative and negation) for $p, q \in\left\{0, \frac{1}{2}, 1\right\}$ by $p \wedge q:=\min \{p, q\}, p \vee q:=$ $\max \{p, q\}$ and $\neg p:=1-p$. To avoid confusion with the standard logic and to emphasize the meaning in our approach of the three logical values $1, \frac{1}{2}$, and 0 , we will call them proved, open and disproved, respectively. Equipped with the threevalued logic we define the following relations between intervals $I=\left[x_{0}, x_{1}\right], J=$ $\left[y_{0}, y_{1}\right] \in \mathcal{I}(\mathbb{R})$

$$
I<J:=\left\{\begin{array}{lll}
\text { proved } & \text { if } x_{1}<y_{0}, \\
\text { disproved } & \text { if } y_{1} \leq x_{0}, \\
\text { open } & \text { otherwise, }
\end{array} \quad I \leq J:= \begin{cases}\text { proved } & \text { if } x_{1} \leq y_{0}, \\
\text { disproved } & \text { if } y_{1}<x_{0}, \\
\text { open } & \text { otherwise. }\end{cases}\right.
$$

We will also need a function which translates the three-valued logic to the standard two-valued logic. We define for $v \in\{$ proved, disproved, open $\}$

$$
\operatorname{can} \_\operatorname{claim}(v):= \begin{cases}\text { true } & \text { if } v=\text { proved } \\ \text { false } & \text { otherwise. }\end{cases}
$$

Again an easy induction argument proves the following proposition.

Proposition 7.2. Assume $p\left(x_{1}, \ldots, x_{n}\right)$ is a sentence built out of the logical operators $\wedge, \vee, \neg$ and relation operators $<, \leq$. Then for any intervals $I_{1}, \ldots, I_{n}$

$$
\text { can_claim }\left(p\left(I_{1}, \ldots, I_{n}\right)\right) \Rightarrow p\left(x_{1}, \ldots, x_{n}\right)
$$

for any numbers $x_{1}, \ldots, x_{n}$ such that $x_{i} \in I_{i}$ for $i=1, \ldots, n$.

\section{Proof of Theorem 1.1}

Algorithms and data structures used in our computer assisted proof are presented in this section in a PASCAL-like style with comments embedded between $(*$ and $*)$. The standard dot notation is used to extract components of compound structures. REAL denotes the type representing a fixed set of representable numbers $\hat{\mathbb{R}} \subset \mathbb{R}$. Minimum and maximum of $x, y \in \mathbb{R}$ are denoted respectively by $x \wedge y$ and $x \vee y$.

Let $i \in\{2,3\}$. Our basic structures are

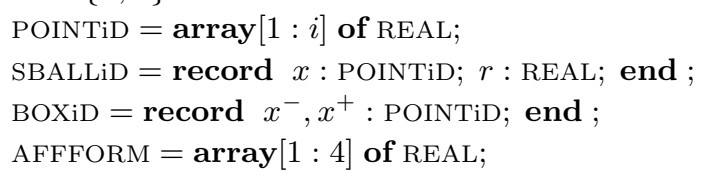


The structures POINT2D and POINT3D are used to represent vectors in $\mathbb{R}^{2}$ and $\mathbb{R}^{3}$. If $x, y$ are two structures of type POINT3D, then $l(x, y)$ will denote the line through these points i.e., $l(x, y) \subset \mathbb{R}^{3}$. The structures SBALL2D and SBALL3D are used to represent balls in $\mathbb{R}^{2}$ and $\mathbb{R}^{3}$ in the supremum metric with $x$ and $r$ denoting respectively the center and the radius. Given $b$ a variable of type SBALL2D(SBALL3D), the actual ball in $\mathbb{R}^{2}\left(\mathbb{R}^{3}\right)$ represented by $b$ will be denoted by $|b|$. The structures BOX2D and BOX3D are used to represent rectangles in $\mathbb{R}^{2}$ and cuboids in $\mathbb{R}^{3}$ with $x^{-}, x^{+}$denoting the corners with minimal and maximal coordinates respectively. If $c$ is a variable of type BOX2D or Box3D, then $|c|$ will denote the actual rectangle in $\mathbb{R}^{2}$ or cuboid in $\mathbb{R}^{3}$ represented by $c$. The structure AFFFORM is used to represent an affine form in $\mathbb{R}^{3}$. If $\xi$ is of type AFFFORM, then $\xi(x):=\xi[1] x_{1}+\xi[2] x_{2}+\xi[3] x_{3}-\xi[4]$ for $x=\left(x_{1}, x_{2}, x_{3}\right) \in \mathbb{R}^{3}$ will denote the value of the associated affine form at $x$, $|\xi|:=\left\{x \in \mathbb{R}^{3} \mid \xi(x)=0\right\}$ will stand for the associated affine plane in $\mathbb{R}^{3}$ and $n_{\xi}$ will denote the normal vector to $|\xi|$.

The structure

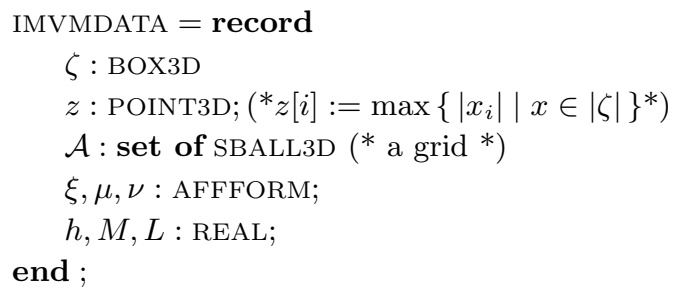

collects all data needed to compute the intermediate map. It includes $\zeta$, the domain in which all estimates are valid, $\xi$, the surface approached, $h$, the step of the numerical method chosen for this domain, $M$ and $L$, the estimates of the vector field and the Lipschitz constant and two coordinate forms $\mu, \nu$ used to efficiently represent the values.

We will say that the structure $\Delta$ of type IMVMDATA is well set for the vector field $V$ if $\Delta . M \geq \sup \left\{\|V(x)\|_{\text {sup }}|x \in| \Delta . \zeta \mid\right\}, \Delta . L \geq \sup \left\{\left\|d_{x} V\right\|_{\text {sup }}|x \in| \Delta . \zeta \mid\right\}$ and $\|\varphi(s, x)-\langle\Phi\rangle(s, x)\|_{\text {sup }} \leq E_{\Delta . \zeta}^{\Phi}(s)$ for $x \in|\Delta . \zeta|_{s}$ and $s>0$ (here $E_{\Delta . \zeta}^{\Phi}$ is the polynomial introduced in [19]). Since the Lorenz vector field is polynomial, it is straightforward to find the appropriate upper bounds for $L$ and $M$ and by Theorem 6.1 the required error estimate is always satisfied if $E_{\Delta . \zeta}^{\Phi}$ is taken as in [19]. Therefore without loss of generality we may assume that in the sequel the structure $\Delta$ is always well set up for the Lorenz vector field.

The combinatorial multivalued maps are stored as sets of pairs of the form (argument, value), such that for any two different pairs their first elements (arguments) are different. To optimize the algorithms it was convenient to appropriately code the values to avoid long lists. For the same purpose we have chosen two different structures to represent the (argument,value)-pairs for the intermediate multivalued maps and for the final multivalued map.

The structure IAVPAIR is used to represent the argument-value pair of the intermediate map. The argument is stored as a 3-dimensional ball in the supremum metric. The value is stored by providing the minimal and maximal values of the $\mu$ and $\nu$ forms. The actual value is the collection of boxes with $\mu, \nu$ coordinates of their centers within the given limits. The intermediate map is stored as a list of argument-value pairs.

IAVPAIRrecord

$b$ :SBALL3D; (* argument*) 


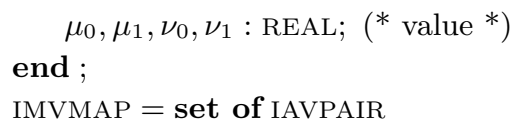

If $\mathcal{F}$ is a variable of type IMVMAP, then we define the combinatorial multivalued map $\mathcal{F}_{\Delta}: \operatorname{dom} \mathcal{F}:=\{q . b \mid q \in \mathcal{F}\} \rightarrow \Delta \cdot \mathcal{A}$ by $\operatorname{dom} \mathcal{F}_{\Delta}:=\{|q . b| \mid q \in \mathcal{F}\}$ and

$$
\mathcal{F}_{\Delta}(q . b):=\left\{c \in \Delta . \mathcal{A} \mid q \cdot \mu_{0} \leq \Delta . \mu(c) \leq q . \mu_{1}, q . \nu_{0} \leq \Delta . \nu(c) \leq q . \nu_{1}\right\} .
$$

The structure AVPAIR is used to represent the argument-value pair of the Poincaré map. The Poincaré map itself is stored as a list of argument-value pairs.

AVPAIRrecord $\alpha, \omega:$ SBALL2D; end ;

MVMAP $=$ set of AVPAIR

Since the value $\omega$ is a strongly representable set over a fixed grid $\mathcal{G}_{\gamma_{2}}^{2}$, we can associate with a variable $\Pi$ of type MVMAP a combinatorial multivalued map $\Pi_{\mathcal{G}_{\gamma_{2}}^{2}}$ given by

$$
\operatorname{dom} \Pi_{\mathcal{G}_{\gamma_{2}}^{2}}:=\{q . \alpha \mid q \in \Pi\}, \Pi_{\mathcal{G}_{\gamma_{2}}^{2}}(q . \alpha):=\mathcal{G}_{\gamma_{2}}^{2}(q . \omega) .
$$

In the algorithms presented in this section it is assumed that all arithmetic expressions evaluated within the upper or can_claim functions are evaluated in the interval arithmetic and using Łukasiewicz logic. Note that all arguments in such expressions are always representable numbers not intervals. Thus, it is assumed they are converted to intervals just before the expression is evaluated. Also, the value returned by the function upper is always a representable number. This means that we do not need variables of type interval. Intervals are used only in course of evaluating expressions as temporary objects. It should be emphasized that expressions other than those mentioned above are evaluated in a representable arithmetic. This does not influence the rigor of proofs based on these algorithms, because the results of expressions evaluated this way are used only as choices. Any other choice would be legitimate, but it could increase the chances that the algorithm would fail. In what follows it is assumed that $V$ denotes a fixed rational vector field in $\mathbb{R}^{3}, \sigma_{\text {sup }}(D V(x))$ is the logarithmic norm of $D V(x)$ corresponding to the supremum norm, all variables $\Delta$ are set for the vector field $V, \Phi$ is the classic fourth order Runge-Kutta method (see [4], [14]). Furthermore, we assume that $\mathcal{G}_{\gamma_{2}}^{2}, \mathcal{G}_{\gamma_{3}}^{3}$ are two fixed grids in $\mathbb{R}^{2}$ and $\mathbb{R}^{3}$ respectively.

We begin the description of the algorithms we use with the following one, which finds an upper bound on the image of a 3 -dimensional box under the $h$-translation map of the flow.

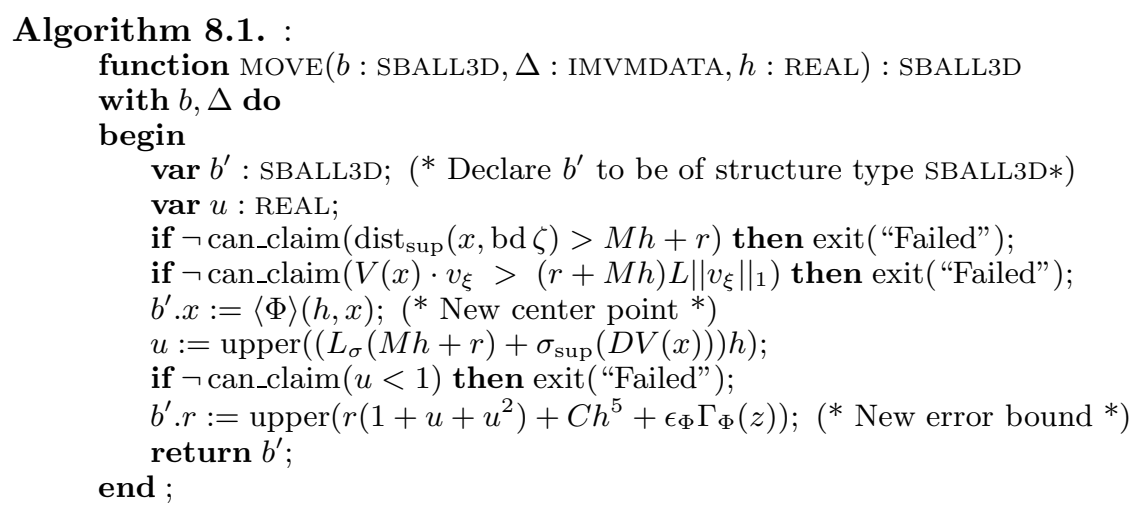


Proposition 8.2. If Algorithm 8.1 does not return "Failed", then $\varphi(h,|b|) \subset\left|b^{\prime}\right|$ and $\varphi([0, h],|b|) \subset|\Delta . \zeta|$. Moreover, $V(y) \cdot v_{\xi}>0$ for any $y \in \varphi([0, h],|b|)$.

Proof. If the algorithm does not return "Failed", then $\operatorname{dist}_{\text {sup }}(x, \mathrm{bd}|\Delta . \zeta|)>M h+r$. Thus the assumptions of Lemma 6.2 are satisfied, and hence the first statement of the proposition is true. To prove the inequality take $y \in \varphi([0, h],|b|)$. Then $\|y-x\| \leq r+M h$ and $\|V(y)-V(x)\| \leq L(r+M h)$. Thus, under the assumption that the algorithm does not return "Failed", we get

$$
\begin{aligned}
& V(y) \cdot v_{\xi}=V(x) \cdot v_{\xi}-(V(x)-V(y)) \cdot v_{\xi} \\
& \quad \geq V(x) \cdot v_{\xi}-\|V(x)-V(y)\|_{\text {sup }}\left\|v_{\xi}\right\|_{1} \geq V(x) \cdot v_{\xi}-L(r+M h)\left\|v_{\xi}\right\|_{1}>0 .
\end{aligned}
$$

The next algorithm is used to compute the intermediate multivalued map. It is assumed that on entry the grid $\mathcal{A}_{0}$ contains the domain in which the map is to be computed. For the most part, when computing the intermediate multivalued maps, we use a fixed step Runge-Kutta method. However, in order to get the enclosure of the Poincaré map it is necessary to adjust steps in such a way that at the moment of crossing the section we have first the whole ball enclosing the point on trajectory close to the section but entirely below the section and in the next step the whole ball above the section. Moreover, we want the last step as small as possible in order to minimize the final size of the multivalued map enclosing the Poincare map. Thus, the last two steps i.e., the one before and during the crossing, are determined by means of the following two functions

function SELAPPRH : REAL; function SELJUMPH : REAL;

The computations of these steps need not be rigorous, because the steps are just choices. A bad choice may cause Algorithm 8.3 to fail or produce an inefficient enclosure. The algorithm used to find the possible optimal steps is just the standard bisection method with some safety margin to minimize the risk that Algorithm 8.3 fails. Since the algorithm is well known, we do not include it here. Note that on exit the structure $\mathcal{F}$ contains the computed multivalued map and the structure $\Delta . \mathcal{A}$ represents the domain for the next multivalued map.

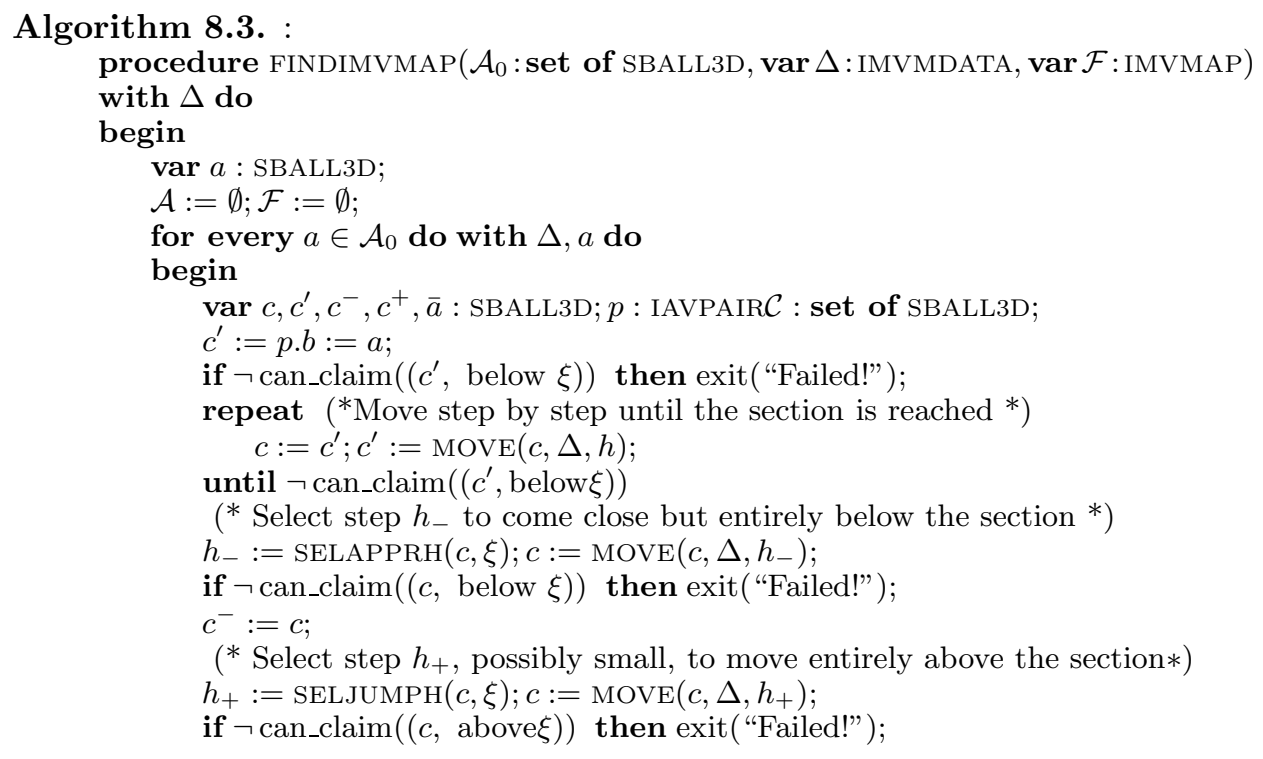




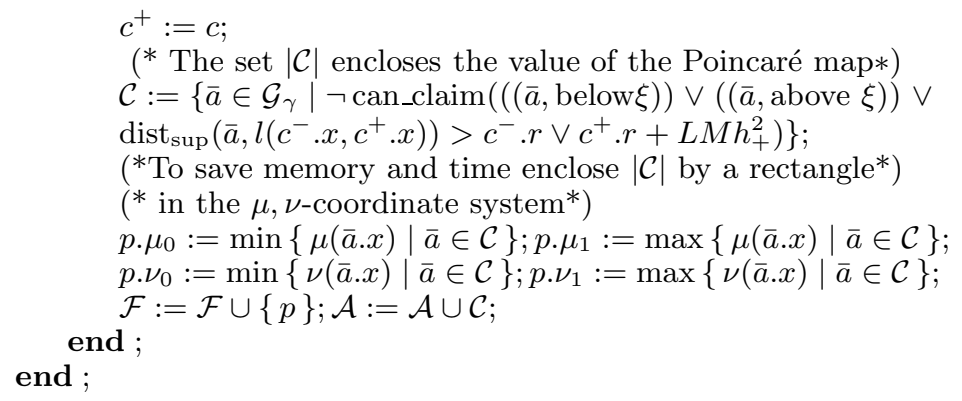

Theorem 8.4. Assume Algorithm 8.3 stops and does not return "Failed". Then on return $\operatorname{dom}\left\lfloor F_{\Delta}\right\rfloor=\left|\mathcal{A}_{0}\right|$ precedes $|\Delta . \xi|$ and

$$
\begin{gathered}
\tilde{\varphi}_{|\Delta . \xi|}\left(\left|\mathcal{A}_{0}\right|\right) \subset|\Delta . \zeta|, \quad \varphi_{|\Delta . \xi|}^{0}\left(\left|\mathcal{A}_{0}\right|\right) \subset|\Delta . \mathcal{A}|, \\
\varphi_{|\Delta . \xi|}^{0}(x) \in\left\lfloor\mathcal{F}_{\Delta}\right\rfloor(x) \text { for every } x \in \operatorname{dom}\left\lfloor\mathcal{F}_{\Delta}\right\rfloor .
\end{gathered}
$$

Proof. We will write $\xi$ instead of $\Delta . \xi$ and we put $\Xi:=|\xi|$. Assume the algorithm stops and does not return "Failed". Let $q$ be a fixed element of $\mathcal{A}_{0}$. Let $K_{q}$ denote the number of calls to function MOVE with variable $a=q$. Since the algorithm stops, $K_{q}<\infty$. Let $b_{0}^{q}:=q$ and for $n=1,2, \ldots, K_{q}$ let $h_{n}^{q}$ denote the last argument in the $n$-th consecutive call of MOVE and $b_{n}^{q}$ the value returned. For $n=0,1, \ldots, K_{q}$ set $H_{n}^{q}:=\left|b_{n}^{q}\right|, W_{n}^{q}:=\varphi\left(\left[0, h_{n}^{q}\right], H_{n-1}^{q}\right)$ and $t_{n}^{q}:=h_{1}^{q}+h_{2}^{q}+\cdots h_{n}^{q}$. Also put $W_{q}:=W_{1}^{q} \cup \ldots \cup W_{K_{q}}^{q}, W:=\bigcup\left\{W_{q} \mid q \in \mathcal{A}\right\}, t_{q}:=t_{K_{q}}^{q}$ and $T:=\max \left\{t_{q} \mid q \in \mathcal{A}\right\}$. It follows from Proposition 8.2 and the definition of $W$ that $\varphi\left(h_{n}^{q}, H_{n-1}^{q}\right) \subset H_{n}^{q}$ for $n=1, \ldots, K_{q}, W \subset|\Delta . \zeta|$ and

$$
V(y) \cdot n_{\xi}>0 \text { for any } y \in W .
$$

An easy induction argument shows that for any $n=1, \ldots, K_{q}$,

$$
\varphi\left(t_{n}^{q}, H_{0}^{q}\right) \subset H_{n}^{q}, \quad \varphi\left(\left[0, t_{n}^{q}\right], H_{0}^{q}\right) \subset W_{1}^{q} \cup \ldots \cup W_{n}^{q} \subset \Delta . \zeta .
$$

Property (17) and Lemma 6.4 imply that $\Xi$ is a local section in $W$. Now, $H_{0}^{q}$ lies below $\Xi$ and $H_{K_{q}}^{q}$ lies above $\Xi$, because otherwise the algorithm would have returned "Failed". Thus, by (18), also $\varphi\left(t_{K_{q}}, H_{0}^{q}\right)$ lies above $\Xi$. Consequently $H_{0}^{q} \subset \operatorname{dom} \varphi_{\Xi}^{0}$ and

$$
0<t_{\Xi}^{0}(x) \leq t_{q} \leq T \text { for } x \in H_{0}^{q}, \quad \varphi\left(\left[0, t_{\Xi}^{0}(x)\right], x\right) \subset W \text { for } x \in H_{0}^{q} .
$$

Property (19) shows that $\operatorname{dom} \mathcal{F}_{\Delta}=\bigcup\left\{H_{0}^{q} \mid q \in P\right\}=\left|\mathcal{A}_{0}\right| \subset E(W, \Xi)$ and $\operatorname{dom} \mathcal{F}_{\Delta}$ precedes $\Xi$.

Since $W \subset|\Delta . \zeta|$, the first inclusion in (15) follows immediately from (19). Let $\mathcal{C}_{q}$ denote the value associated with the variable $\mathcal{C}$ when $a$ takes value $q$ and let $\bar{r}_{q}:=\max \left(b_{K_{q}-1} . r, b_{K_{q}} \cdot r\right)+L M h_{K_{q}}^{2}$. Then

$$
\Xi \cap B_{\sup }\left(\left[b_{K_{q}-1} \cdot x, b_{K_{q}} \cdot x\right], \bar{r}_{q}\right) \subset\left|\mathcal{C}_{q}\right| \cap|\Delta \cdot \mathcal{A}| \subset\left|\mathcal{F}_{\Delta}(q . b)\right| .
$$

Now take $x \in \operatorname{dom} \mathcal{F}_{\Delta}$. Then $x \in H_{0}^{q}$ for some $q \in \mathcal{A}$ and $\varphi_{\Xi}^{0}(x)=\varphi(t, y)$ for some $y \in H_{K_{q}-1}$ and $t \in\left[0, h_{K_{q}}\right]$. It follows from Lemma 6.3 and (20) that

$$
\varphi_{\Xi}^{0}(x) \in \Xi \cap B_{\text {sup }}\left(\left[b_{K_{q}-1} . x, b_{K_{q}} \cdot x\right], \bar{r}_{q}\right) \subset\left|\mathcal{F}_{\Delta}(q . b)\right| \cap|\Delta . \mathcal{A}| .
$$

This, in particular, proves the second inclusion in (15) and since (21) applies to any $q \in \mathcal{A}$ such that $x \in H_{0}^{q}$ we get from the definition of $\left\lfloor\mathcal{F}_{\Delta}\right\rfloor$ that $\varphi_{\Xi}^{0}(x) \in\left\lfloor\mathcal{F}_{\Delta}\right\rfloor(x)$. Thus (16) is proved. 
The next algorithm is used to compose two consecutive multivalued maps. Exactly speaking, it computes only an upper bound on the composition, determined by the $\mu, \nu$ forms. Finding the exact composition would require searching lists, which is rather expensive.

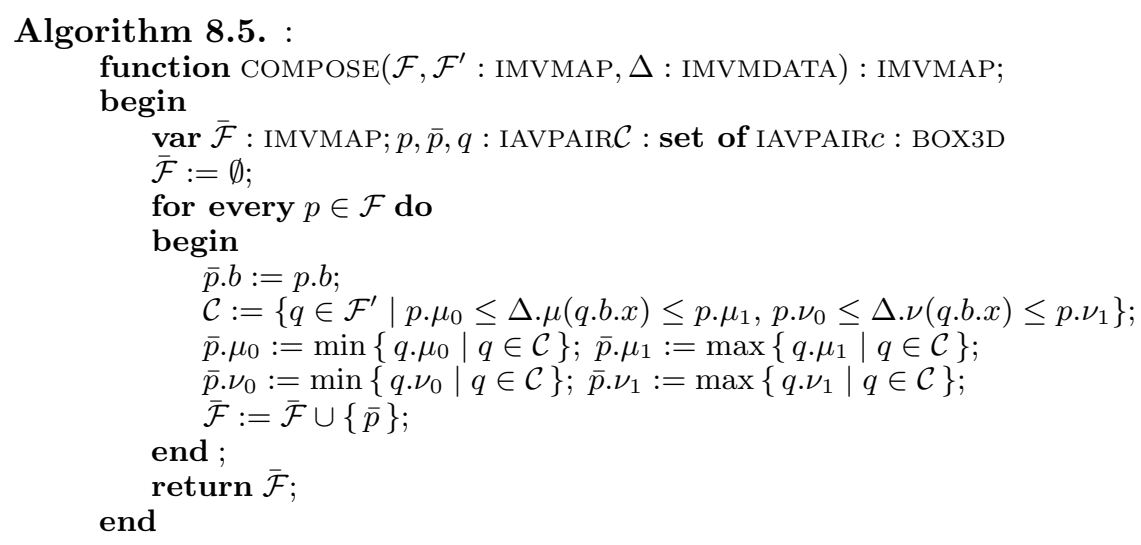

One can easily verify the following proposition.

Proposition 8.6. If $\overline{\mathcal{F}}$ denotes the value returned by function COMPOSE, then the domains of $\overline{\mathcal{F}}_{\Delta^{\prime}}$ and $\mathcal{F}_{\Delta}$ coincide and $\mathcal{F}_{\Delta^{\prime}}^{\prime}\left(\mathcal{F}_{\Delta}(x)\right) \subset \overline{\mathcal{F}}_{\Delta^{\prime}}(x)$ for any $x \in \operatorname{dom} \overline{\mathcal{F}}_{\Delta^{\prime}}$.

Our next algorithm computes a multivalued enclosure of the Poincaré map. The Poincaré section is chosen to be a subset of the plane $\left\{\left(x_{1}, x_{2}, x_{3}\right) \mid x_{3}=R-2\right\}$ which actually is the standard choice for the Lorenz system.

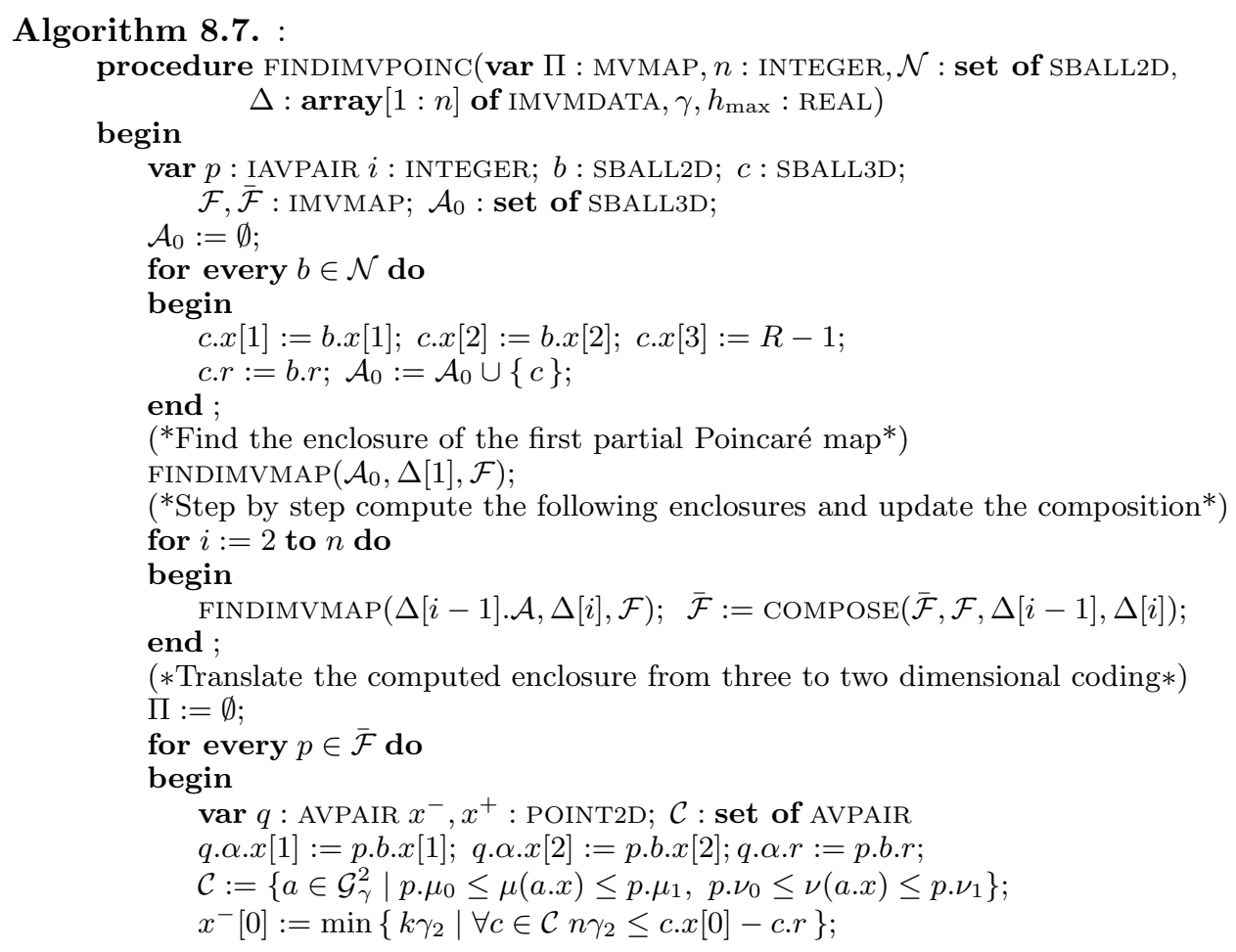


end ;

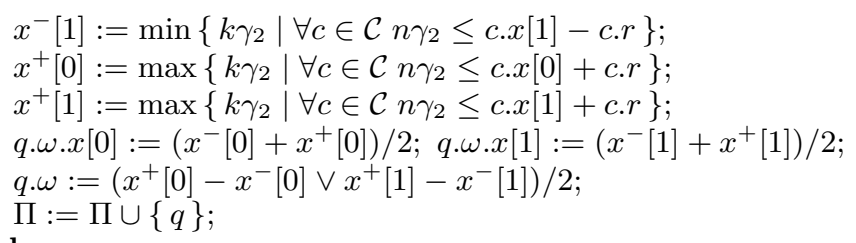

Theorem 8.8. Assume Algorithm 8.7 is called with its arguments satisfying the following two conditions

$$
\begin{gathered}
|\mathcal{N}| \subset|\Theta| \subset|\Delta[n] . \xi| \text { and }|\Delta[n] . \xi| \text { is a local section in }|\Delta[1] . \zeta|, \\
|\Delta[i] \cdot \zeta| \cap \Theta=\emptyset \quad \text { for } i=2, \ldots, n-1,
\end{gathered}
$$

where $\Theta$ is a given compact set. If the algorithm stops and does not fail, then $N:=|\mathcal{N}| \subset \operatorname{dom} \varphi^{\Theta},\left.\varphi^{\Theta}\right|_{N}$ is continuous and $\varphi^{\Theta}(x) \in\left\lfloor\Pi_{\mathcal{G}_{\gamma_{2}}^{2}}\right\rfloor(x)$ for $x \in N$, where $\Pi$ denotes the multivalued map returned by Algorithm 8.7.

Proof. For $i=1,2, \ldots, n$, let $\mathcal{A}_{i-1}$ denote the value of the first argument in the $i$-th call to Findimvmap and let $\mathcal{A}_{n}:=\{q \cdot \omega \mid q \in \Pi\}$. The construction of Algorithm 8.7, Theorem 8.4 and conditions (22), (23) imply that the sets $A_{i}:=\left|\mathcal{A}_{i}\right|$ and $\Xi:=|\xi|$ satisfy the assumptions of Lemma 5.2. Thus $N \subset \operatorname{dom} \varphi^{\Theta}$ and $\operatorname{dom} \varphi_{\mid N}^{\Theta}$ is continuous. Put $\Xi_{i}:=|\Delta[i] . \xi|$. Let $\mathcal{F}_{i}$ denote the combinatorial multivalued map associated with the $i$-th consecutive value of variable $\overline{\mathcal{F}}$ and $\Delta[i]$. An easy induction argument based on property (16) of Proposition 8.4 and Theorem 8.6 shows that

$$
\varphi_{\Xi_{i-1} \mid A_{i-1}}^{0} \circ \cdots \circ \varphi_{\Xi_{1} \mid A_{0}}^{0}(x) \in\left\lfloor\mathcal{F}_{i}\right\rfloor(x) .
$$

Thus, by property (9) of Lemma 5.1 we have

$$
\left.\varphi^{\Theta}\right|_{N}(x)=\varphi_{\Xi_{n} \mid A_{n-1}}^{0} \circ \cdots \circ \varphi_{\Xi_{1} \mid A_{0}}^{0}(x) \in \Xi \cap\left\lfloor\mathcal{F}_{n}\right\rfloor(x) \subset\left\lfloor\Pi_{\mathcal{G}_{\gamma_{2}}^{2}}\right\rfloor(x) .
$$

Our last algorithm is used to verify if an isolating block was found and to construct the corresponding exit set.

Algorithm 8.9. :

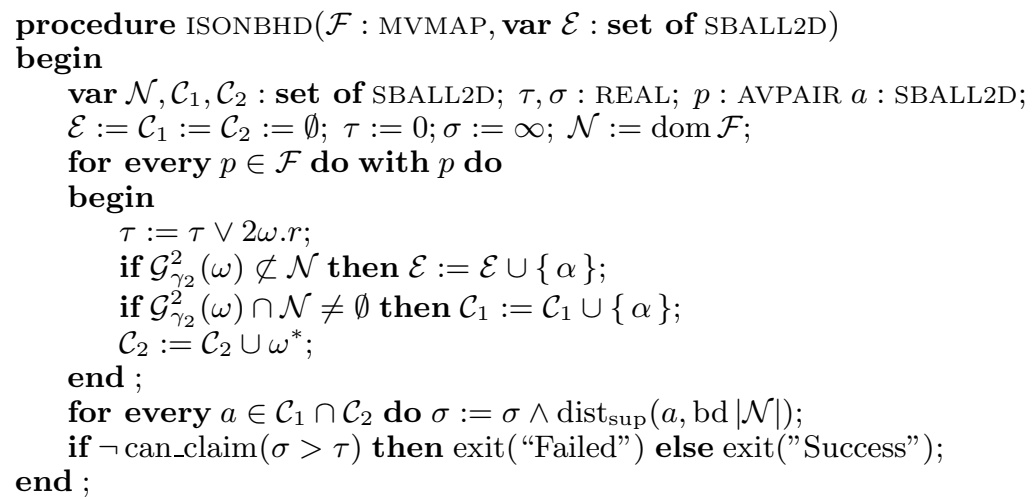

Theorem 8.10. If the above algorithm succeeds, then $N:=|\operatorname{dom} \mathcal{F}|$ is an isolating block for $F:=\lfloor\mathcal{F}\rfloor$ and on return the value of the variable $\mathcal{E}$ represents the set $E:=|\mathcal{E}|$ such that the pair $(N, E)$ is an index pair for $F$ in $N$. 
Proof. It follows from the construction of $\mathcal{C}_{1}, \mathcal{C}_{2}$ that $\mathcal{F}^{*-1}(\mathcal{N}) \cap \mathcal{F}(\mathcal{N})^{*}=\mathcal{C}_{1} \cap \mathcal{C}_{2}$ and $\mathcal{E}=\mathcal{N} \backslash \mathcal{F}^{-1}(\mathcal{N})$. Thus

$$
\operatorname{dist}_{\text {sup }}\left(\left|\mathcal{F}^{*-1}(\mathcal{N}) \cap \mathcal{F}(\mathcal{N})^{*}\right|, \operatorname{bd}|\mathcal{N}|\right) \geq \operatorname{dist}_{\text {sup }}\left(\left|\mathcal{C}_{1} \cap \mathcal{C}_{2}\right|, \operatorname{bd} N\right)=\sigma>\tau=\operatorname{diam} \mathcal{F}
$$

and the thesis follows from Theorem 4.3.

Remark 8.11. If $\mathcal{N} \subset \mathcal{G}_{\gamma_{2}}^{2}$, where $\gamma_{2}$ is a representable number, then the computation of $\operatorname{dist}_{\text {sup }}(a$, bd $|\mathcal{N}|)$ may be performed exactly but may be lengthy if $\mathcal{N}$ is large. In our application to the Lorenz system we used the fact that $\mathcal{N}$ was taken as a covering of a set of 4 parallelograms (see the next section). In such a case an interval estimate from below of this distance may be obtained by means of Lemma 6.5. We then compared with $\tau$ this estimate instead of the exact distance.

We are finally ready to turn our attention to the Lorenz equations. First observe that the Lorenz equations are equivariant with respect to the symmetry

$$
\rho: \mathbb{R}^{3} \ni\left(x_{1}, x_{2}, x_{3}\right) \rightarrow\left(-x_{1},-x_{2}, x_{3}\right) \in \mathbb{R}^{3} .
$$

Let $\varphi$ be the flow generated by the Lorenz equations (1) with $(s, R, q)=(45,54$, 10). Take $\bar{M}_{10}, \bar{M}_{11}$ to be the two parallelograms as indicated in Figure 2 (see [14] for exact definitions of $\left.\bar{M}_{10}, \bar{M}_{11}\right)$. For $i, j=0,1$ let

$$
\begin{aligned}
& \left.\mathcal{M}_{1, i}:=\left\{a \in \mathcal{G}_{1500 / 2^{20}} \mid \neg \text { can_claim(dist } \operatorname{dup}_{\text {sup }}\left(a, \bar{M}_{1, i}\right)\right)>0\right\}, \\
& \mathcal{M}_{0, i}:=\left\{\rho(a) \mid a \in \mathcal{M}_{1,1-i}\right\}, \mathcal{N}:=\mathcal{M}_{00} \cup \mathcal{M}_{01} \cup \mathcal{M}_{10} \cup \mathcal{M}_{11}, \\
& M_{i, j}:=\left|\mathcal{M}_{i, j}\right|, \quad N_{0}:=M_{00} \cup M_{01}, N_{1}:=M_{10} \cup M_{11}, \quad N:=N_{0} \cup N_{1} \text {, } \\
& \Xi:=\left\{\left(x_{1}, x_{2}, x_{3}\right) \in \mathbb{R}^{3} \mid x_{3}=53\right\}, \quad \Theta:=[2,17] \times[-2,4] \times\{53\} .
\end{aligned}
$$

Lemma 8.12. The Poincaré map is well defined on $N$, i.e. $N \subset \operatorname{dom} \Psi_{\Theta}$ and $\left.\Psi_{\Theta}\right|_{N}$ is continuous. Moreover, if $E$ denotes the shaded region in Figure 2 (more precisely: if $E:=|\mathcal{E}|$, where $\mathcal{E}$ is the output produced by Algorithm 8.9), then $(N, E)$ is an index pair for $\Psi_{\Theta}$, the Alexander-Spanier cohomology of the index pair is given by

$$
H^{i}(N, E)= \begin{cases}\mathbb{Z}^{8} & \text { for } i=1, \\ 0 & \text { otherwise }\end{cases}
$$

and the associated index map is

$$
I_{(N, E)}=\left[\begin{array}{cccccccc}
1 & 0 & 0 & 0 & 1 & 0 & 0 & 0 \\
1 & 0 & 0 & 0 & 1 & 0 & 0 & 0 \\
0 & 1 & 0 & 0 & 0 & 1 & 0 & 0 \\
0 & 1 & 0 & 0 & 0 & 1 & 0 & 0 \\
0 & 0 & 1 & 0 & 0 & 0 & 1 & 0 \\
0 & 0 & 1 & 0 & 0 & 0 & 1 & 0 \\
0 & 0 & 0 & 1 & 0 & 0 & 0 & 1 \\
0 & 0 & 0 & 1 & 0 & 0 & 0 & 1
\end{array}\right] .
$$

Proof. Take an arithmetic complying with the 64-bit IEEE standard (see [29]). Take $\Phi$ to be the classic 4-th order Runge-Kutta method for (1) (see [4], [14]). Let $n=23$. For $i=1,2, \ldots, n$, initialize the variables $\Delta_{j}[i] \cdot \zeta, \Delta_{j}[i] \cdot \xi, \Delta_{j}[i] . \mu, \Delta_{j}[i] . \nu$ as in Tables 1-4 in the Appendix of [14] (The precise binary values of these numbers may be fetched from http://www.ii.uj.edu.pl/mrozek/lorenz.html or delivered via e-mail on request - send e-mail to mrozek@ii.uj.edu.pl). Take $\mathcal{G}^{3}:=\mathcal{G}_{\frac{300}{2^{20}}}^{3}, \mathcal{G}^{2}:=$ 
$\mathcal{G}_{\frac{1500}{2^{20}}}^{2}, h_{\max }:=\frac{1000}{2^{20}}$ and start Algorithm 8.7 twice for $j=0,1$. In both cases it stops and does not fail. Let $\Pi_{j}(j=0,1)$ be the multivalued map returned by Algorithm 8.7. Put $\Pi:=\Pi_{0} \cup \Pi_{1}$ and extend this map to a map $\Pi$ on $\mathcal{N}$ using the symmetry $\rho$. It is now an easy exercise to verify that the assumptions of Theorem 8.8 are satisfied. Thus $N \subset \operatorname{dom} \Psi_{\Theta}, \Psi_{\Theta \mid N}$ is continuous and $\Psi_{\Theta}(x) \in\lfloor\Pi\rfloor(x)$ for $x \in N$, This shows that $\Psi_{\Theta}$ is a selector of $\lfloor\Pi\rfloor$.

Algorithm 8.9 started with $\mathcal{F}=\Pi$ succeeds and returns $\mathcal{E}$ as in Figure 2. (Though this does not belong to the proof, the final values of variables $\sigma$ and $\tau$ we obtained were 0.058 and 0.046 respectively.) Thus the rest of the assertion follows from Theorem 8.10 and Theorem 4.2.

Proof of Theorem 1.1. Let $N, E$ be as in Lemma 8.12. Using the form of the index map in $N$ given by (24) and arguing as in the proof of Theorem 2.3 in [12] one can easily check that the assumptions of Theorem 2.1 are satisfied. The thesis follows.

\section{ACKnowledgments}

Both authors gratefully acknowledge Luca Dieci's advice regarding the numerical computations necessary in this work. It is a pleasure of the second author to thank Colin Sparrow for several conversations held many years ago which turned out to be crucial in the choice of the Lorenz equations as an illustration of the presented method.

\section{REFERENCES}

[1] U. Ascher, R.M. Mattheij and D.R. Russell, Numerical Solution of Boundary Value Problems for ODEs, Prentice-Hall, Englewood Cliffs, N.J., 1988. MR 90h:65120

[2] Xinfu Chen, Lorenz Equations, Part III: Existence of Hyperbolic Sets, preprint 1995.

[3] C. Conley, Isolated Invariant Sets and the Morse Index, CBMS Regional Conf. Ser. Math., no 38, AMS, Providence, R.I., 1978. MR 80c:58009

[4] E. Hairer, S.P. Nørsett and G. Wanner, Solving Ordinary Differential Equations I, Nonstiff Problems, Springer-Verlag, Berlin Heidelberg 1987. MR 87m:65005

[5] B. Hassard, J. Zhang, S. Hastings, and W. Troy, A computer proof that the Lorenz equations have "chaotic" solutions, Appl. Math. Letter 7 (1994), 79-83. MR 96d:58082

[6] S.P. Hastings and W.C. Troy, A shooting approach to the Lorenz equations, Bulletin (New Series) of the American Mathematical Society 27(1992) 298-303. MR 93f:58150

[7] T. Kaczyński and M. Mrozek, Conley index for discrete multivalued dynamical systems, Topology ES its Appl., 65(1995), 83-96. MR 97d:54066

[8] R.J. Lohner, Computation of Guaranteed Enclosures for the Solutions of Ordinary Initial and Boundary Value Problems, in: Computational Ordinary Differential Equations, J.R. Cash, I. Gladwell Eds., Clarendon Press, Oxford, 1992. CMP 96:12

[9] J. Łukasiewicz, O logice trójwartościowej (On three-valued logic), Ruch Filozoficzny 5(1920), 169-170.

[10] K. Mischaikow, The structure of isolated invariant sets, Contemporary Mathematics, C. McCord ed.,AMS, (1993), 269-290. MR 94k:58083

[11] K. Mischaikow, The Conley index theory: some recent developments, CIME Lectures, preprint.

[12] K. Mischaikow and M. Mrozek, Isolating neighborhoods and Chaos, Jap. J. Ind. ES Appl. Math., 12, 1995, 205-236. MR 96e:58104

[13] K. Mischaikow and M. Mrozek, Chaos in Lorenz equations: a computer assisted proof, Bull. $A M S$, 32(1995), 66-72. MR 95e:58121

[14] K. Mischaikow and M. Mrozek, Chaos in Lorenz equations: a computer assisted proof. Part II: Details, preprint CDSNS95-222. 
[15] K. Mischaikow, M. Mrozek and A. Szymczak, Chaos in Lorenz equations: a computer assisted proof. Part III: The classical case, in preparation.

[16] J. Moser, Stable and Random Motions in Dynamical Systems, Princeton Univ. Press (1973). MR 56:1355

[17] M. Mrozek, Leray Functor and the Cohomological Conley Index for Discrete Dynamical Systems, Transactions of the American Mathematical Society 318(1990) 149-178. MR 90f:34076

[18] M. Mrozek, Topological invariants, multivalued maps and computer assisted proofs in dynamics, Computers \& Mathematics, 32(1996),83-104. MR 97h:58144

[19] M. Mrozek, Rigorous error analysis of numerical algorithms via symbolic computations, $J$. Symb. Comp., 22(1996) 435-458. CMP 97:06

[20] J. Munkres, Elements of Algebraic Topology, Addison-Wesley, 1984. MR 85m:55001

[21] A. Neumaier, The wrapping effect, ellipsoid arithmetic, stability and confidence regions, Computing Supplementum, 9 (1993), 175-190.

[22] A. Neumaier, Global, rigorous and realistic bounds for the solution of dissipative differential equations Part I: Theory, Computing 52 (1994), 315-336. MR 95f:65098

[23] T. Rage, A. Neumaier, and C. Schlier, Rigorous verification of chaos in a molecular model, Physical Rev. E, 50 (1994), 2682-2688.

[24] K.P. Rybakowski, The Homotopy Index and Partial Differential Equations, Springer Verlag, Berlin Heidelberg 1987. MR 89d:58025

[25] M.R. Rychlik, Lorenz attractors through Šil'nikov-type bifurcation. Part I, Ergodic Theory E Dynamical Systems, 10(1989), 793-821. MR 92f:58103

[26] S. Smale, Differentiable dynamical systems, Bull. AMS 73 (1967), 747-817. MR 37:3598

[27] H. Spreuer, E. Adams, On the strange attractor and transverse homoclinic orbits for Lorenz equations, J. Math. Anal. and Appl. 190(1995), 329-360. MR 96h:58119

[28] P. Zgliczyński, Computer assisted proof of chaos in the Rössler equations and in the Hénon map, Nonlinearity 10 (1997), 243-252. CMP 97:07

[29] The IEEE Standard for Binary Floating-Point Arithmetic, ANSI/IEEE Std 754,1985.

Center for Dynamical Systems and Nonlinear Studies, School of Mathematics, Georgia Institute of Technology, Atlanta, Georgia 30332-0001

E-mail address: mischaik@math.gatech.edu

Center for Dynamical Systems and Nonlinear Studies, School of Mathematics, Georgia Institute of Technology, Atlanta, Georgia 30332-0001

Current address: Instytut Informatyki, Uniwersytet Jagielloński, Kraków, Poland

E-mail address: mrozek@ii.uj.edu.pl 